Research paper

\title{
Fast multipole method applied to Lagrangian simulations of vortical flows
}

\author{
Túlio R. Ricciardi ${ }^{\mathrm{a}}$, William R. Wolf ${ }^{\mathrm{a}, *}$, Alex M. Bimbato ${ }^{\mathrm{b}}$ \\ a University of Campinas, Campinas, SP, 13083-860, Brazil \\ b São Paulo State University, Guaratinguetá, SP, 12516-410, Brazil
}

\section{A R T I C L E I N F O}

\section{Article history:}

Received 28 April 2016

Revised 3 April 2017

Accepted 8 April 2017

Available online 13 April 2017

\section{Keywords:}

Vortex dynamics

Fast multipole method

Discrete vortex method

Trefftz plane

Temporal shear layer

\begin{abstract}
A B S T R A C T
Lagrangian simulations of unsteady vortical flows are accelerated by the multi-level fast multipole method, FMM. The combination of the FMM algorithm with a discrete vortex method, DVM, is discussed for free domain and periodic problems with focus on implementation details to reduce numerical dissipation and avoid spurious solutions in unsteady inviscid flows. An assessment of the FMM-DVM accuracy is presented through a comparison with the direct calculation of the Biot-Savart law for the simulation of the temporal evolution of an aircraft wake in the Trefftz plane. The role of several parameters such as time step restriction, truncation of the FMM series expansion, number of particles in the wake discretization and machine precision is investigated and we show how to avoid spurious instabilities. The FMM-DVM is also applied to compute the evolution of a temporal shear layer with periodic boundary conditions. A novel approach is proposed to achieve accurate solutions in the periodic FMM. This approach avoids a spurious precession of the periodic shear layer and solutions are shown to converge to the direct Biot-Savart calculation using a cotangent function.
\end{abstract}

(c) 2017 Elsevier B.V. All rights reserved.

\section{Introduction}

We solve canonical flows with practical applications in physics and engineering using the discrete vortex method, DVM, which is a Lagrangian method that avoids computational mesh generation. In this method, the Navier-Stokes equations are solved in the vorticity form by the discretization of the vorticity field using $N$ discrete elements, which are transported with the local flow velocity. As shown by Chorin [1], the convection and diffusion processes appearing in the vorticity equation can be solved separately in the DVM and the calculation of the non-linear term is avoided through application of the material derivative, associated to Lagrangian methods. The convection term may be solved using a potential vortex model which does not introduce numerical diffusivity. In this context, spurious numerical instabilities may appear when the DVM is employed for the solution of inviscid flow problems modeled by vortex sheets. In order to regularize the solution of potential vortices, other models may be employed adding numerical diffusion to the vorticity field. Recently, the DVM has been applied to solve two dimensional problems including airfoil leading edge separation [2,3], unsteady motion of a pitching airfoil [4] and cylinder wake instability [5]. A review of the method can be seen in Barba et al. [6] and Aref [7].

\footnotetext{
* Corresponding author.

E-mail address: wolf@fem.unicamp.br (W.R. Wolf).
} 
In order to solve the velocity field using the DVM, one must compute the interactions among discrete vortex particles which are governed by the Biot-Savart law. This leads to a computational cost proportional to $\mathrm{O}\left(N^{2}\right)$ which may be reduced by the implementation of a fast algorithm. Here, the fast multipole method, FMM, is chosen since it reduces the computational cost from $\mathrm{O}\left(N^{2}\right)$ to $\mathrm{O}(N)$ when $N$ is sufficiently large and a hierarchical multi-level algorithm is used. The method was initially proposed by Greengard and Rokhlin [8], and later optimized by Carrier et al. [9] for the solution of potential fields. The FMM has been applied in the literature to accelerate the solution of several problems involving potential flows [10], acoustic and electromagnetic scattering [10-13] and aero-acoustics [14,15]. An efficient implementation of the FMM was proposed by Gumerov and Duraiswami for the simulation of vortical flows [16].

Fast algorithms have been also applied in combination with particle methods for the solution of problems with periodic boundary conditions [17,18]. For instance, Yokota and Barba, and Yokota and Obi employed the periodic FMM to study isotropic turbulence and homogeneous shear flows, respectively [19,20]. Sakajo and Okamoto [21] present an approach in which an exponential mapping transforms the periodic DVM function into a rational function. Then, these authors employ a fast method [22] to simulate the Kelvin-Helmholtz instability problem. Recently, Marple et al. [23] developed a fast algorithm to solve multiphase flows and applied the method for the solution of Stokes flows in periodic channels of arbitrary geometry.

In the present work, the coupled FMM-DVM algorithm is employed to simulate the time evolution of an aircraft wake and a periodic shear layer. The first problem represents the formation of wing tip vortices which appear in the wake of an airplane. Aerodynamic wakes of large aircraft and their induced velocity may cause serious hazards to smaller aircraft, affecting the take-off and landing operations in airports. A simplified but representative model of the problem is the evolution of a vortex sheet in the Trefftz plane, as explained in details by Smith [24]. The second problem is relevant in the context of several problems of engineering and physics involving ocean mixing, cloud formation, multi-phase flow, combustion and jet flows (Murray et al. [25], Smyth and Moum [26], Herrmann [27]). In viscous flow problems, physical dissipation will overcome the numerical dissipation that is introduced in the computation of the convective step via time marching scheme and vortex regularization [28]. However, in the study of hydrodynamic instabilities which may appear in inviscid flows, numerical dissipation can be an issue and accurate solutions are required.

This study focus on the implementation details of the FMM-DVM algorithm with the aim to reduce numerical dissipation in unsteady inviscid flows. We evaluate the pros and cons from coupling both the FMM and DVM in order to avoid spurious solutions. To do so, we analyze the role of temporal discretization and aspects of regularization of vortex models. We also provide an assessment of the parameters which control the errors of the FMM, for instance, the refinement level and the number of terms in the series expansions. Discretization effects are discussed for the solution of the Trefftz plane problem showing that a convergence to the truly inviscid solution, without instabilities, can be achieved. Previous work has showed the presence of spurious instabilities in the calculation of this problem for quasi-inviscid solutions (Fink and Soh [29], Krasny [30-32], Abid and Verga [33]). Lastly, a periodic problem is solved with a FMM implementation which reduces truncation error using balanced periodic boundary conditions to avoid a spurious precession of the infinite shear layer.

\section{Numerical methodology}

\subsection{Discrete vortex method}

The discrete vortex method solves the Navier-Stokes equations in the vorticity form. For an incompressible Newtonian fluid, the 2D vorticity transport equation in non-dimensional variables is given by

$$
\frac{\partial \omega}{\partial t}+\mathbf{u} \cdot \nabla \omega=\frac{1}{R e} \nabla^{2} \omega,
$$

where $\mathbf{u}=u \hat{\mathrm{i}}+v \hat{\mathrm{j}}$ is the velocity vector, $\omega=\nabla \times \mathbf{u}$ is the z-vorticity component and Re is the Reynolds number. Chorin [1] proposed a numerical solution of Eq. (1) solving separately the inviscid and viscous terms in two fractional steps. The first step considers the flow to be inviscid, i.e., $\operatorname{Re} \rightarrow \infty$, leading to

$$
\frac{\partial \omega}{\partial t}+\mathbf{u} \cdot \nabla \omega=\frac{\mathrm{D} \omega}{\mathrm{D} t}=0,
$$

while the second step solves the viscous effects

$$
\frac{\partial \omega}{\partial t}=\frac{1}{R e} \nabla^{2} \omega
$$

The calculation of the inviscid term requires the solution of the Biot-Savart law, which computes the velocity field due to the vortex interactions. For a two-dimensional problem, the Biot-Savart law is written as

$$
\mathbf{u}(\mathbf{x}, t)=\frac{1}{2 \pi} \int_{S^{\prime}} \frac{\omega\left(\mathbf{x}^{\prime}, t\right) \times\left(\mathbf{x}-\mathbf{x}^{\prime}\right)}{\left|\mathbf{x}-\mathbf{x}^{\prime}\right|^{2}} d S^{\prime}
$$




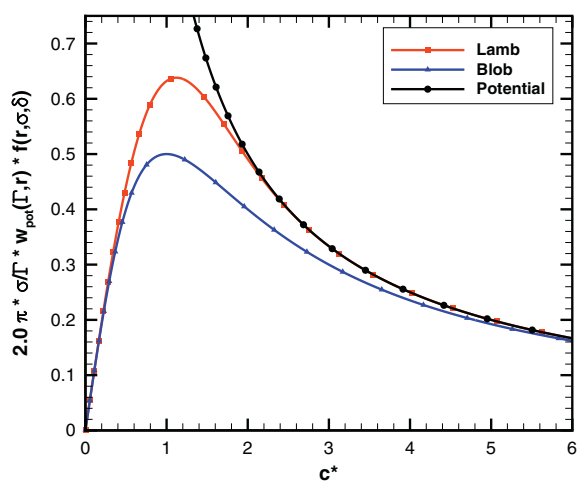

Fig. 1. Velocity profiles for different vortex models.

In complex notation, the velocity induced by a potential vortex $j$ at a point $k$ is given by Eq. (5) for a vortex with circulation defined by $\Gamma>0$ in clockwise sense

$$
w_{k}=u_{k}-\mathrm{i} v_{k}=\mathrm{i} \frac{\Gamma_{j}}{2 \pi z_{k j}},
$$

with the complex distance given by $z_{k j}=\left(x_{k}-x_{j}\right)+\mathrm{i}\left(y_{k}-y_{j}\right)$.

In the present work, we want to investigate the effects of numerical diffusivity which are present even in the inviscid part of the DVM. This artificial diffusion is introduced either by the regularization of the potential vortex or through the time marching scheme [28]. These are important aspects related to the combination of the DVM with the fast multipole method.

In order to overcome the singularity for $r \rightarrow 0$, where $r=\left|z_{k j}\right|$ is the distance between points $k$ and $j$, a model for the vorticity distribution $\omega$ should be implemented. One of the most used models with non-singular velocity field is the LambOseen vortex, which has a fixed second-order Gaussian core $\sigma$ to represent the core diffusion. For a radial distance $r$, its induced velocity is given by:

$$
w_{k}=\mathrm{i} \frac{\Gamma_{j}}{2 \pi z_{k j}}\left[1-\exp \left(-\frac{r^{2}}{\sigma^{2}}\right)\right]=\mathrm{i} \frac{\Gamma_{j}}{2 \pi z_{k j}}(1-f(r, \sigma)) .
$$

Another regularization model is the vortex blob, given by Eq. (7). It was proposed by Krasny [30] and it is based in the Hilbert transform with a conjugate Poisson kernel, where $\delta$ is an arbitrary parameter. This model converges to the potential vortex when $\delta \rightarrow 0$, for the induced velocity given by

$$
w_{k}=\mathrm{i} \frac{\Gamma}{2 \pi z_{k j}}\left(\frac{1}{1+\frac{\delta^{2}}{r^{2}}}\right)=\mathrm{i} \frac{\Gamma_{j}}{2 \pi z_{k j}} f(r, \delta) .
$$

Other alternatives to smooth out the vorticity distribution are shown by Chorin [1], who truncates the velocity profile in regions very close to the vortex; by Holm et al. [34] who proposed a model using an Euler-alpha regularization; or the application of a Rankine vortex, where the velocity varies linearly inside the viscous core.

The velocity profile of a potential vortex, which is representative of a truly inviscid flow, is presented in Fig. 1 . In the same figure, auto-similar velocity profiles are shown for the blob and Lamb-Oseen vortex models which are used to represent physical aspects in a realistic vortex core. Solutions are presented in terms of the non-dimensional core, $c^{*}$, which is given by $\frac{r}{\sigma}$ and $\frac{r}{\delta}$ for the Lamb-Oseen and blob vortex models, respectively. One can observe that for $\delta=\sigma$ the latter is more dissipative than the former.

In order to understand the influence of the time marching scheme on the numerical diffusion, we simulate the dynamics of a large Gaussian vortical structure discretized with $30 \mathrm{k}$ particles. In this problem, the interaction among all vortex particles creates a rotational motion of the entire structure as shown by Lewis [35]. The particles are randomly distributed at $x_{i}$ and $y_{i}$, following a Gaussian shape with standard deviation $\tilde{\sigma}=0.1$. Previous work involving problems with the absence of viscosity or boundaries employ the computation of the Hamiltonian, an operator that indicates the energy conservation in the system, as a metric to be used for time accuracy $[28,30,32]$. The Hamiltonian is measured as

$$
H=\frac{1}{2 \pi} \sum_{i=1}^{N} \sum_{j=1}^{N} \Gamma_{i} \Gamma_{j} \log \left(\left|z_{i j}\right|\right) \quad \text { when } i \neq j .
$$

Simulations are performed using a viscous core $\sigma=0.001$ and total circulation $\Gamma=10.0$, which is equally divided among all $N$ particles. Following the definition of a vortical structure, the radial component of the velocity should be null. For a dimensionless time $t^{*}=0.5$, Fig. 2 shows the vorticity distribution for the first and fourth order Runge-Kutta schemes, RK1 


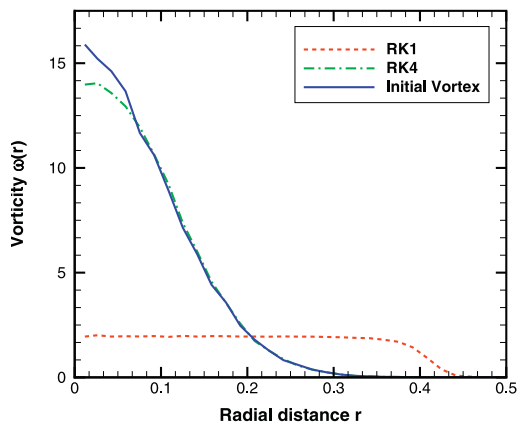

(a) 50 steps, $\Delta \mathrm{t}=0.01, \sigma=$ $0.001, \Gamma=10.0$.

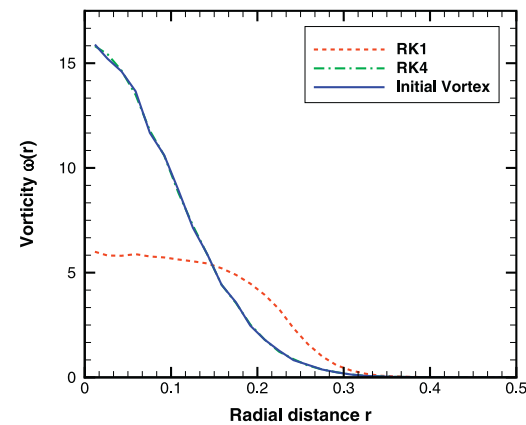

(b) 500 steps, $\Delta \mathrm{t}=0.001, \sigma=$ $0.001, \Gamma=10.0$.

Fig. 2. Analysis of the time marching schemes.

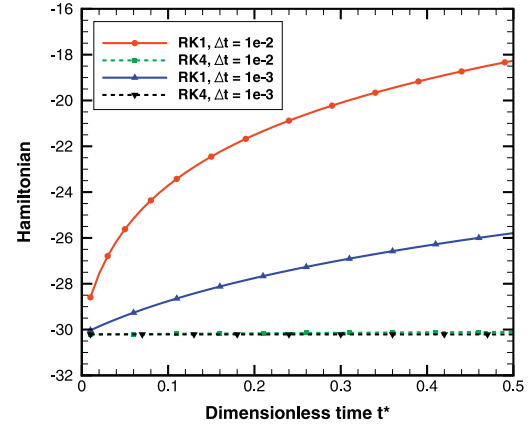

(c) Hamiltonian, $\Gamma=10$.

and RK4, respectively. In Fig. 2(a) and (b), the solutions are compared to the initial shape of the large vortex and they show that a larger time step may introduce numerical diffusion. One can also see from these figures that a low accuracy in the time marching method tends to spread the particles. Fig. 2(c) presents the calculation of the Hamiltonian of the system and one can observe that the scattering of the particles introduced by the numerical diffusion of the low order scheme reduces the kinetic energy of the entire system. Based on the present analysis, the following computations in this work are performed using the RK4 time marching scheme with the aim of reducing the numerical dissipation in the solutions.

\subsection{Fast multipole method}

The fast multipole method is applied to accelerate the solution of $N$-body problems. In the FMM algorithm, a computational domain, selected as a square box in $2 \mathrm{D}$ or a cubical box in $3 \mathrm{D}$, involves all the discrete elements. This computational domain which is considered to be at level zero of refinement in the FMM tree $(\ell=0)$ can be further divided to create smaller boxes at higher refinement levels $(1 \leq \ell \leq L)$ that define far and near-field clusters of elements. The far-field interactions are responsible for reducing the computational cost of the method towards $\mathrm{O}(N)$ by the application of a divide-and-conquer strategy. The fast method is not applicable among groups of elements close to each other and, hence, the near-field interactions still require direct evaluations of the problem. Therefore, for each box, at each refinement level, the FMM requires the calculation of two sets of lists which indicate the boxes that are in the near and far-field regions. They are called neighbor and interaction lists, respectively. For more details on the FMM, the authors refer to Greengard and Rokhlin, [8], Carrier et al. [9], Wolf [10] and Nishimura [36]. The main equations used in the FMM implementation are shown in the present section for completeness.

The potential $\phi$ at a distant observer $z_{0}$ from a source with unitary strength placed at $z_{S}$ is given by the Green's function

$$
\phi_{s}\left(z_{0}\right)=G\left(z_{s}, z_{0}\right)=\log \left(z_{0}-z_{s}\right)
$$

for the observer and source position, respectively, $z_{0}=x_{0}+\mathrm{i} y_{0}$ and $z_{s}=x_{s}+\mathrm{i} y_{s}$.

In the DVM-FMM, mathematical relations are used to cluster a set of $N_{b}$ sources (vortex particles) with circulation $\Gamma_{s}$ inside a box $b$ centered at $z_{b}$ at the finest level of refinement $L$. The sources are clustered in the particle-to-multipole step (P2M), Eq. (10), which applies a Taylor series expansion of Eq. (9). One can truncate the series after $p$ terms in order to obtain the multipole expansions, given by $\mathcal{M}_{k}(b, L)$, with $k=0 \ldots p$.

$$
\mathcal{M}_{0}(b, L)=\sum_{s=1}^{N_{b}} \Gamma_{s} \quad \text { and } \quad \mathcal{M}_{k}(b, L)=-\sum_{s=1}^{N_{b}} \frac{\Gamma_{s} z^{k}}{k} .
$$

The multipole expansions represent the intensity of the cluster of sources inside box $b$ at level of refinement $L$ and $z$ is the complex distance from the source $s$ to the box $b$ center, $z=z_{s}-z_{b}$.

The multipole expansions can be further grouped into larger clusters of vortex particles in the multipole-to-multipole step (M2M). This recursive step uses children boxes $b_{\mathcal{C}}$ at level $\ell+1$, for $0<\ell<L-1$. The new clusters are formed in the parent boxes $B$ at level $\ell$. The M2M step is given by

$$
\mathcal{M}_{0}(B, \ell)=\sum_{\mathcal{C}=1}^{4} \mathcal{M}_{0}\left(b_{\mathcal{C}}, \ell+1\right)
$$


and, for $k=1$ to $p$ :

$$
\mathcal{M}_{k}(B, \ell)=\sum_{\mathcal{C}=1}^{4}\left\{\sum_{m=1}^{k}\left[\left(\begin{array}{c}
k-1 \\
m-1
\end{array}\right) \mathcal{M}_{m}\left(b_{\mathcal{C}}, \ell+1\right) \cdot z^{k-m}\right]-\mathcal{M}_{0}\left(b_{\mathcal{C}}, \ell+1\right) \frac{z^{k}}{k}\right\} .
$$

In the equation above, the term $\left(\begin{array}{c}k-1 \\ m-1\end{array}\right)$ is the binomial coefficient and the complex distance from the center of the child box $b_{\mathcal{C}}$ to its parent box $B$ center is given by $z=z_{b_{\mathcal{C}}}-z_{B}$

Once the multipole expansions are computed, it is possible to calculate the multipole-to-local step (M2L). This step provides the induced velocity at the observer cluster $b$ from all the 27 well-separated source clusters $b_{s}$ which are in the interaction list of $b$. These operations are performed for boxes at refinement level $\ell$, as

$$
\mathcal{L}_{0}^{(M 2 L)}(b, \ell)=\sum_{s=1}^{27}\left[\mathcal{M}_{0}\left(b_{s}, \ell\right) \cdot \log (-z)+\sum_{k=1}^{p}\left(\mathcal{M}_{k}\left(b_{s}, \ell\right) \frac{(-1)^{k}}{z^{k}}\right)\right]
$$

and, for $m=1$ to $p$ as

$$
\mathcal{L}_{m}^{(M 2 L)}(b, \ell)=\sum_{s=1}^{27}\left\{-\frac{\mathcal{M}_{0}\left(b_{s}, \ell\right)}{m \cdot z^{m}}+\frac{1}{z^{m}} \sum_{k=1}^{p}\left[\left(\begin{array}{c}
m+k-1 \\
k-1
\end{array}\right) \mathcal{M}_{k}\left(b_{s}, \ell\right) \frac{(-1)^{k}}{z^{k}}\right]\right\} .
$$

In the equations above $z=z_{b_{s}}-z_{b}$ and the term $\mathcal{L}(b, \ell)$ is the local representation of the far-field multipole expansions at level $\ell$ computed in the center of box $b$. The local representations can be transfered from the centers of the parent boxes at level $\ell-1$ to those of their children at level $\ell$. This step is called local-to-local (L2L) and, for $m=0$ to $p$, it is given by

$$
\mathcal{L}_{m}^{(L 2 L)}(b, \ell)=\sum_{k=m}^{p}\left(\begin{array}{c}
k \\
m
\end{array}\right) \cdot \mathcal{L}_{k}(B, \ell-1) \cdot(-z)^{k-m},
$$

where $z=z_{B}-z_{b}$ and $1 \leq \ell \leq L$.

The L2L and M2L steps are coupled in order to add the contribution of clusters at coarser levels to those at the same level. Therefore, the local representation is first computed from the L2L operation, Eq. (15). Then, the M2L operations, EqS. (13) and (14), are performed to account for the interactions of far-field clusters of sources contained in boxes at the same level of refinement. The equation to compute the local expansion $\mathcal{L}_{m}(b, \ell)$ for box $b$ at level $\ell$ is then written as

$$
\mathcal{L}_{m}(b, \ell)=\mathcal{L}_{m}^{(L 2 L)}(b, \ell)+\mathcal{L}_{m}^{(M 2 L)}(b, \ell)
$$

After all the far-field interactions are accounted for in $\mathcal{L}_{m}$, until the finest level $L$, the translation from the local representation within box $b$, centered at $z_{b}$, to the observer particle placed at $z_{0}$, is given by the local-to-particle (L2P) operation as

$$
\phi\left(z_{0}\right)=\sum_{m=0}^{\infty} \mathcal{L}_{m}(b, L) \cdot\left(z_{0}-z_{b}\right)^{m}
$$

\subsection{Coupling of DVM and FMM}

The FMM can be applied to accelerate the convolution step of the DVM, which appears as the solution of the Biot-Savart law. In the DVM, it is necessary to evaluate the velocity field generated by the far-field sources, which can be computed from the derivative of the potential as

$$
w\left(z_{0}\right)^{(F F)}=\frac{\partial \phi\left(z_{0}\right)}{\partial z_{0}}=\sum_{m=1}^{\infty} \mathcal{L}_{m}(b, L) \cdot m \cdot\left(z_{o}-z_{b}\right)^{m-1} .
$$

Since for $m=0$ the expression is null, it is not necessary to evaluate $\mathcal{L}_{0}$ in the previous FMM steps.

After computing the velocity from far-field clusters, it is necessary to evaluate the influence from vortices at adjacent boxes that do not satisfy the separation criterion of the FMM. This calculation is performed using the Biot-Savart law for vortices $n$, which can be inside the 8 neighbor boxes of $b_{\mathcal{C}}$ or box $b_{\mathcal{C}}$ itself, to an observer $z_{0}$ inside $b_{\mathcal{C}}$. As all particles are close to each other, it is necessary to use a regularization function $f(r, \sigma, \delta)$ (Lamb or blob vortex model) in order to obtain a smooth velocity profile in the near-field given by

$$
w\left(z_{0}\right)^{(N F)}=\sum_{j=1}^{9} \sum_{n=1}^{N j} \frac{\Gamma_{n}}{z_{0}-z_{n}} f(r, \sigma, \delta) .
$$

Combining both far and near-field calculations leads to the full velocity field in the original reference system as

$$
w\left(z_{0}\right)=w\left(z_{0}\right)^{(N F)}+w\left(z_{0}\right)^{(F F)},
$$




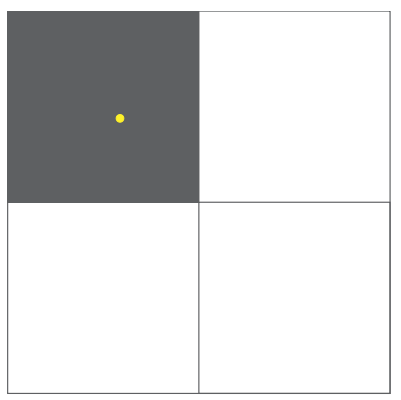

(a) Box at level 1.

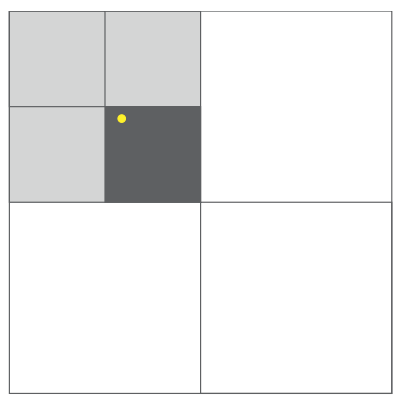

(b) Box at level 2 .

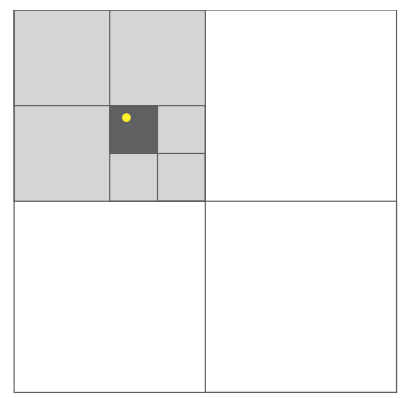

(c) Box at level 3 .

Fig. 3. Efficient way for mapping the location of vortex elements in the multi-level FMM.

where the kernel $\frac{1}{z}$ is solved. For a potential vortex, one should consider Eq. (5) where both the imaginary number $i$ and the constant $\frac{1}{2 \pi}$ are present. Finally, the velocity components are given by

$$
\left[u\left(x_{0}, y_{0}\right), v\left(x_{0}, y_{0}\right)\right]=\frac{1}{2 \pi}\left[\operatorname{Im}\left(w\left(z_{0}\right)\right), \operatorname{Re}\left(w\left(z_{0}\right)\right)\right] .
$$

In the current FMM implementation, we apply an uniform refinement of the initial computational domain at level 0 . Another possibility would be to apply an adaptive refinement approach [9]. However, this other method would require the creation of all the interaction lists every time that refinement is applied increasing pre-processing time. Moreover, the adaptive method would have to deal with the fact that regions that are highly populated with vortices at one time instant can be empty a few steps later and vice-versa. This can be seen as a challenge that appears in the simulation of dynamic problems.

In the uniform refinement approach, the domain is divided until the maximum level, $L$, during the preprocessing step. Whenever a particle leaves the FMM domain or when the density of particles becomes too high, making the near-field computations too expensive, this step is recomputed. This avoids the constant evaluation of the preprocessing, saving computational effort. The creation of boxes in the uniform refinement approach is faster than that in the adaptive one, since it does not depend on the local number of particles. It is only dependent on the number of boxes and maximum refinement level. Also, both interaction and neighbor lists are independent of the domain size, spatial displacement and number of elements, so they do not need to be computed at every time step. Hence, computational cost of pre-processing can be reduced.

Empty boxes can be ignored to reduce computational cost, and they are identified with the mapping of the particles, a step that is computed at every sub-step of the time marching method. A fast way is used here to map the particles and it is based on the genealogical tree of the FMM. Recursively comparing the $x$ and $y$ coordinates of any particle with respect to the center of a box at level $\ell-1$, it is possible to determine the level $\ell$ box which contains the particle. This procedure, based on quadrants, repeats until the finest level is achieved, as shown in Fig. 3. An auxiliary variable counts how many particles are inside the boxes, leading to the list of non-empty boxes.

The kernel of the original FMM is given by the logarithmic function (potential of a singular vortex) and/or its derivatives (velocity field induced by a potential vortex). However, both Lamb-Oseen and blob vortex models have terms that affect the velocity in the near-field and converge to a potential vortex solution in the far-field, which is the region of interest for application of the FMM. In order to choose a suitable vortex model, an initial study of how far is the far-field is necessary for both Lamb-Oseen and blob models. Auto-similar profiles of induced velocity for the two models are presented in Fig. 1, showing that for $r \rightarrow \infty$, the solution of both models converge to the potential one.

However, in the FMM, the regions $r$ of interest for calculations are given by a few box lengths. The convergence of the smoothing parameter $f(r, \sigma)$ in Eq. (6) indicates that the exponential term from the Lamb vortex decays to zero, even for quadruple machine precision, for a box about 9 times the size of the vortex core $\sigma$ (see Fig. 4(a)). The smoothing parameter $f(r, \delta)$ of the vortex blob model proposed by Krasny [30], Eq. (7), slowly converges (in an algebraic sense with $\mathrm{O}\left(\frac{1}{c^{2}}\right)$ ) to the potential solution as observed in Fig. 4(b). The latter shows a slow decay which indicates a non-compact vorticity distribution, being the opposite of the Lamb model. One can see in Fig. 4(c) a comparison of the convergence of smoothing parameters for both vortex models.

With that in mind, we prefer to use the Lamb-Oseen vortex model with the FMM due to its faster exponential decay. Here, based on the results from Fig. 4, and as a generalization for the most restrictive computation using quadruple machine precision, it is imposed that the size of the smallest box in the multi-level FMM, $S_{\ell}$, is at least 9 times the size of the largest vortex core in the domain

$$
S_{\ell}>9 \sigma_{\max } .
$$

This criterion is shown in Fig. 5(a). For double precision, the smallest box, $S_{\ell}$, could be 6 times the size of the Lamb-Oseen vortex viscous core. Therefore, the choice from Eq. (22) does not compromise neither the physics nor the numerical aspects 


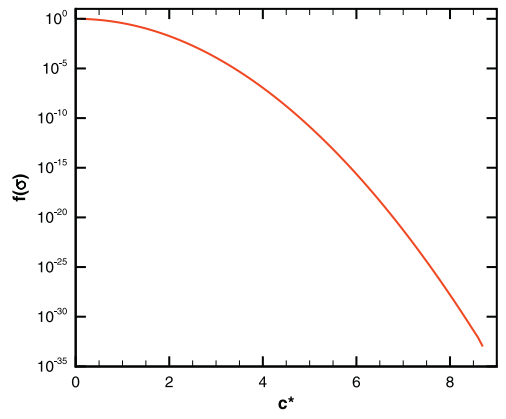

(a) Lamb-Oseen smoothing parameter.

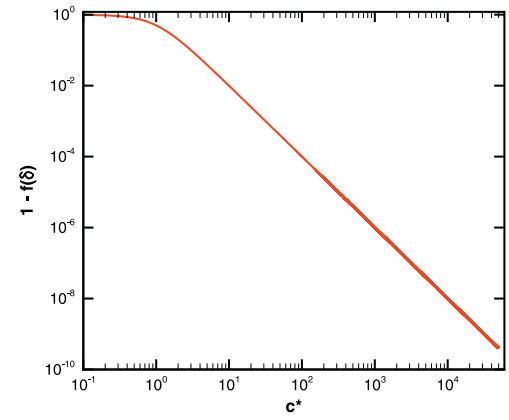

(b) Vortex blob parameter.

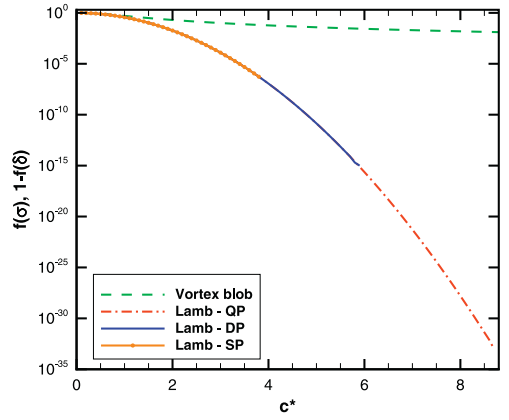

(c) Convergence of the smoothing parameter for different machine precision.

Fig. 4. Comparison between the Lamb-Oseen and vortex blob models.

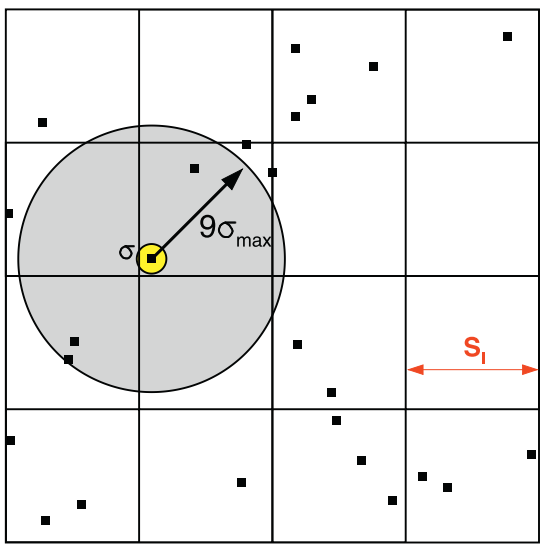

(a) Influence region (in gray color) of the smoothing parameter.

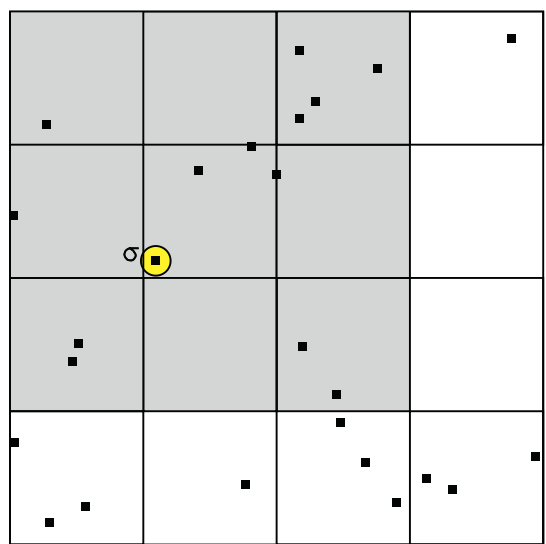

(b) Near-field interactions (in gray color) computed using the Lamb vortex.

Fig. 5. Details on the use of the Lamb-Oseen vortex model applied to the FMM.

of the simulation. In other words, the refinement level is limited based on the Lamb vortex core. A similar observation is provided by Hamilton and Majda [37] who also discuss about the importance of an exponential regularization method for fast convergence of the vorticity field. The present restriction on the smallest box size could be eliminated by using different fast summation methods. For example, Draghicescu and Draghicescu [22] developed a tree-code algorithm using far-field Cartesian Taylor expansions that apply directly to the regularized model.

For practical purposes, the direct calculations performed for particles in neighbor boxes (near-field interactions) are accounted using the Lamb-Oseen vortex while, for far-field FMM interactions, the potential model is employed, Fig. 5(b). Hence, large errors in the induced velocity are introduced if a higher level of refinement is employed to reduce the nearfield cost. The consequences from additional refinements would then be spurious solutions.

\section{Temporal evolution of aircraft wake}

In this section, an assessment of the FMM accuracy is presented through a comparison with the direct solution of the Biot-Savart law. Both methods are applied to compute the temporal evolution of an aircraft wake. This is a very sensitive non-linear problem where accuracy is a critical factor, mainly towards a truly inviscid case with small regularization value of the potential vortex, i.e., $\sigma \rightarrow 0$ in the Lamb vortex model. The role of several parameters such as the number of particles in the wake discretization, refinement level and number of truncation terms in the FMM, and machine precision is investigated.

Since the Trefftz plane is an initial value problem, the initial conditions of the wake have to be defined and, then, its evolution is solved in time. A sketch of the problem is presented in Fig. 6(a) and (b), where the variation of circulation along the span of a finite wing and the tip vortices forming along its wake are shown, respectively. The initial strength of the vortex-sheet is given by the derivative of the circulation along the wingspan, which is determined for an elliptically 


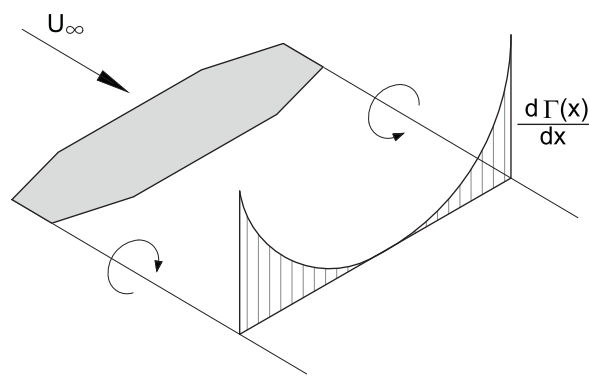

(a) Variation of circulation along the span of a finite wing.

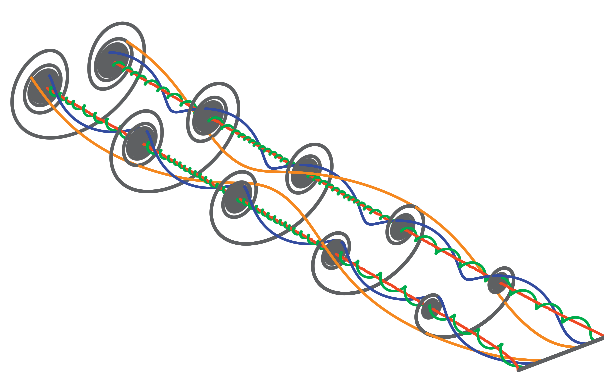

(b) Wing tip vortices forming along the wake.

Fig. 6. Temporal evolution of an aircraft wake modeled in the Trefftz plane.

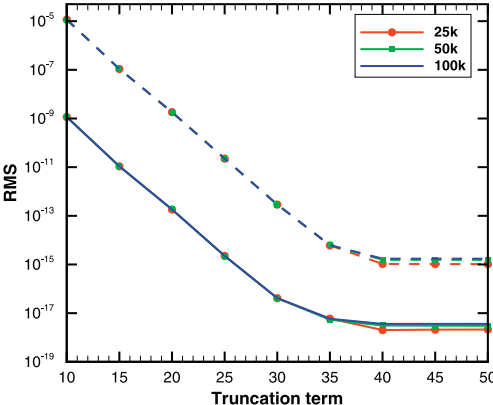

(a) Error due to number of vortex particles.

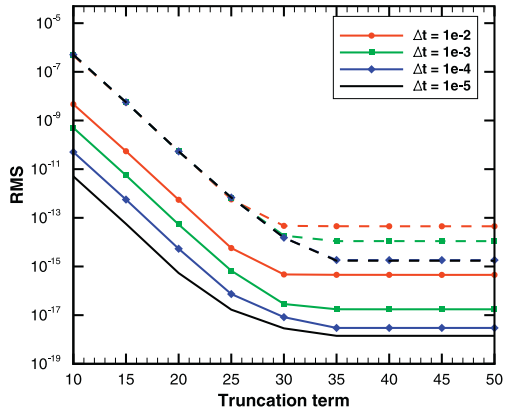

(b) Error due to different time steps.

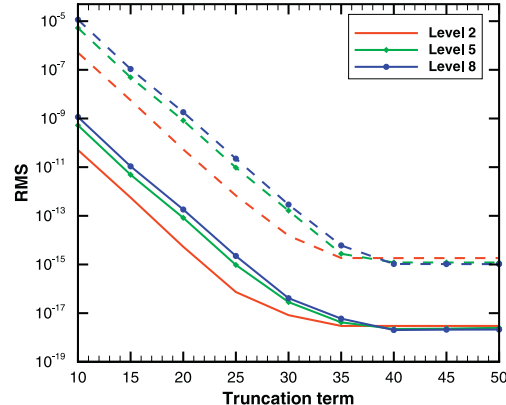

(c) Error due to different refinement levels.

Fig. 7. Assessment of RMS deviation for velocity and position of vortices as a function of series truncation in the FMM.

loaded wing where the circulation varies smoothly by

$$
\frac{\mathrm{d} \Gamma}{\mathrm{d} x}=-\frac{x}{\sqrt{1-x^{2}}} .
$$

In this case, the span is positioned from $-1 \leq x \leq 1$, with a singularity at $|x|=1$. The wake is then partitioned into $N$ segments, concentrated towards the span extremities, and their circulation is given by the integral of Eq. (23) along the length of each segment. Gaussian quadrature with 3 points is applied to integrate the circulation. Other initial conditions can be used according to the lift distribution and one can also simulate the effects of deployed flaps and fuselage, as shown by Krasny [32].

\subsection{Assessment of fast multipole method parameters}

The number of terms used in the FMM series expansion directly impacts the error of the numerical scheme and, therefore, the overall accuracy of the combination between the FMM and DVM. The solutions obtained by the application of the coupled method are compared to those obtained solely by the DVM, which only solves the Biot-Savart law. Fig. 7 shows the error behavior of the FMM as a function of the number of terms retained in the expansion series. Results are presented for the root-mean-square, RMS, deviation in terms of velocity (dashed lines) and vortex position (solid lines) after one convection step. Calculations are performed for $\sigma=1 \times 10^{-3}$.

Fig. 7(a) shows that the error is not affected by the number of vortex particles but only by the terms retained in the FMM series expansions. Results in this figure are obtained for $\Delta t=10^{-4}$ and refinement level 8. In Fig. 7(b), one can see results of the error analysis for FMM refinement level 2 for $25 \mathrm{k}$ vortex particles. An assessment of the influence of the time step, $\Delta t$, on the error is presented for the fourth-order Runge-Kutta time marching scheme. It is possible to see that smaller time steps reduce errors in the FMM computation of both velocity and position. As expected, increasing the number of terms in the FMM series also reduces the error. One can also see that, for all values of time step, the error in position converges to the computer double precision when 35 terms are used in the series. However, the velocity does not converge to the machine precision since several operations in the FMM suffer from truncation errors. Fig. 7(c) presents the error analysis for different levels of refinement of the FMM for a fixed time step of $\Delta t=1 \times 10^{-4}$ and $25 \mathrm{k}$ particles. One can see 


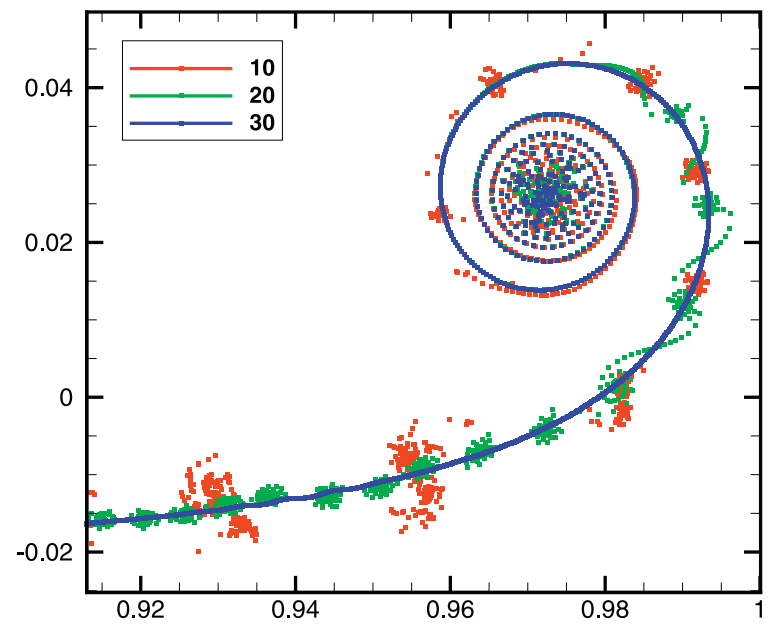

Fig. 8. Analysis of the number of truncation terms in the FMM power series expansions.

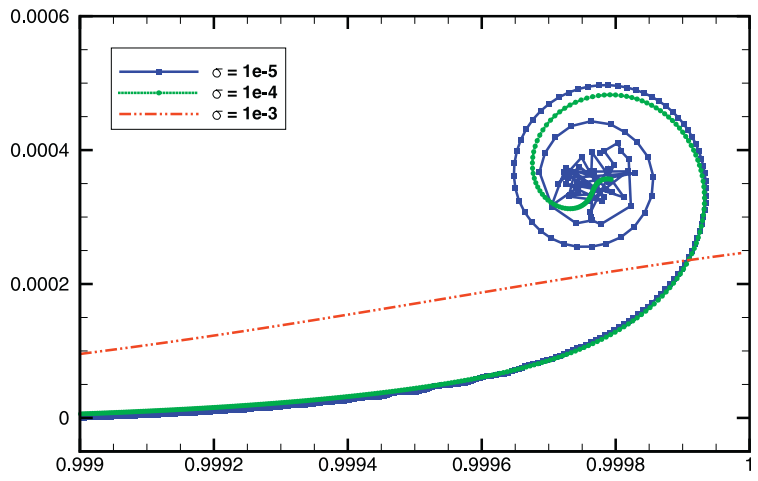

Fig. 9. Roll-up process for different viscous cores, $\sigma$. Solutions obtained with 200,000 vortex particles, RK4, $\Delta t=4 \times 10^{-7}$ and $t=3 \times 10^{-5}$.

that higher levels of refinement produce larger errors when few terms are employed in the FMM series. However, double precision convergence is achieved for 40 truncation terms for all levels of refinement.

\subsection{Vortex sheet roll-up}

In the current study of the Trefftz plane, we solve only the convective operator of the DVM. In this step, numerical dissipation is introduced by the time marching scheme and the vortex regularization. While in viscous flow problems physical dissipation will overcome the numerical dissipation, for inviscid solutions, spurious numerical instabilities will appear. These instabilities need to be carefully handled to avoid their presence. This study focus on the implementation details of the FMM-DVM algorithm with the aim to reduce numerical dissipation in unsteady inviscid flows in order to avoid spurious solutions. The first analysis on this matter refers to the FMM accuracy. Fig. 8 presents the solution of the wing tip vortex roll-up for a different number of terms in the FMM power series expansion. Simulations are integrated for the same instant of time, $t=0.04$, using $25 \mathrm{k}$ particles, $\Delta t=10^{-4}$ and $\sigma=1 \times 10^{-3}$. The red, green and blue dots represent solutions obtained using 10, 20 and 30 terms, respectively. As one can see, the solutions obtained using fewer terms present scattering and secondary roll-up of vortex particles. However, the solution with 30 terms, which provides an error close to machine precision according to Fig. 7, delays the formation of instabilities. In the computations along this work, we employ 40 terms in the FMM series to guarantee the delay in the formation of spurious roll-ups.

Since the FMM allows computations with higher resolutions through discretizations with a larger number of vortex particles along the wake, it is possible to reduce the core $\sigma$ and still maintain a continuous shear layer (the distance between two vortices should still be lower than the viscous core). In this sense, Fig. 9 presents a study of the dynamics of the roll-up for different viscous cores in the Lamb vortex model. Solutions are obtained by the FMM-DVM for 200,000 vortex particles using the RK4 for 75 time steps with $\Delta t=4 \times 10^{-7}$.

Results shown in Fig. 9 demonstrate that smaller viscous cores lead to a faster roll-up, approximating a desired inviscid solution. As one can see in the figure, when $\sigma=1 \times 10^{-3}$, the vortex sheet does not begin the roll-up process and, for $\sigma=1 \times 10^{-5}$, the sheet shows an advanced stage of the roll-up process with a disorganized core. Furthermore, in this last 


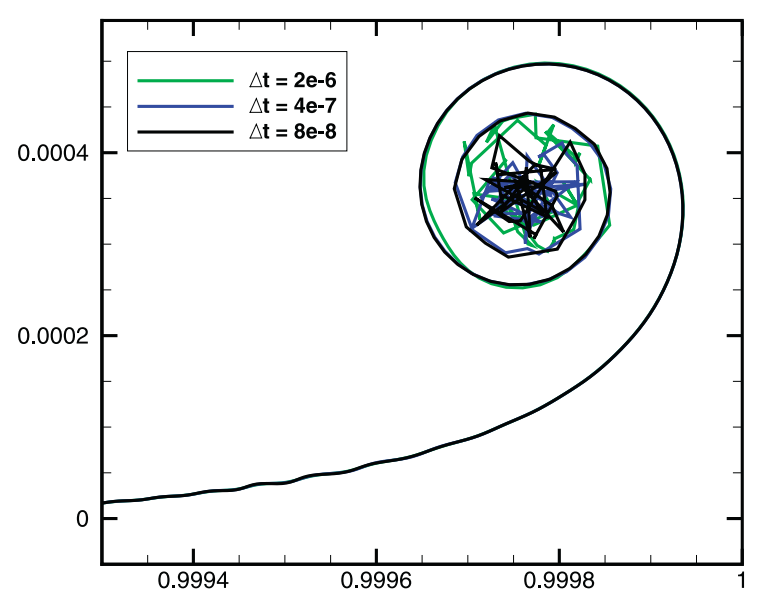

Fig. 10. Time step influence on roll-up process for 200,000 vortex particles, $\sigma=1 \times 10^{-5}$, RK 4 and $t=3 \times 10^{-5}$.
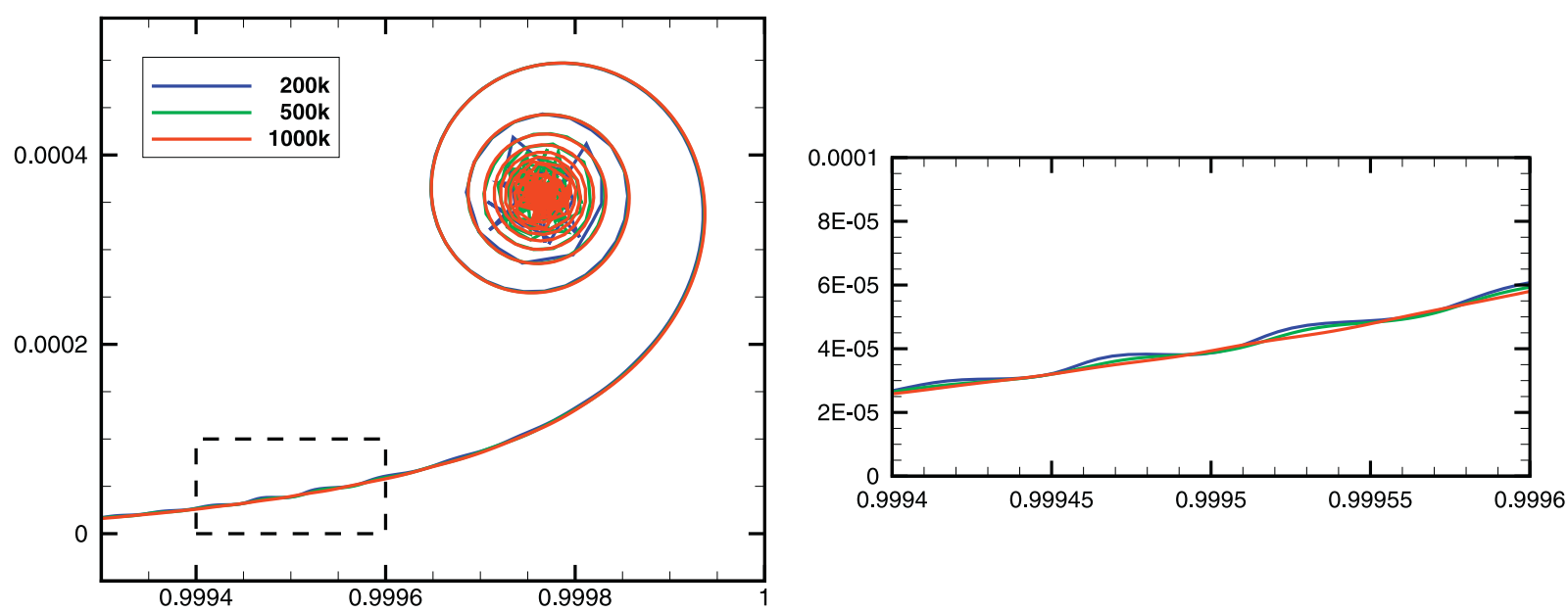

Fig. 11. Influence of number of particles for $\sigma=1 \times 10^{-5}$, RK4, $\Delta t=8 \times 10^{-8}$ and $t=3 \times 10^{-5}$.

case, it is possible to notice a spurious perturbation of the sheet. From now on, we will focus the studies in a vortex wake which is more representative of the inviscid solution and results will be presented for $\sigma=1 \times 10^{-5}$.

As one can observe in Fig. 10, smaller time steps lead to a better development of the roll-up in the wake tip. However, even for $\Delta t=8 \times 10^{-8}$, there is still a chaotic pattern in the core. Furthermore, since the viscous core is very small in the current calculations, the dissipation of the Lamb vortex model is not sufficient to suppress the growth of spurious instabilities in the wake.

The effects of increasing the number of vortex particles are now analyzed for a small time step, $\Delta t=8 \times 10^{-8}$. Fig. 11 shows that a higher resolution leads to overall better results with more layers in the roll-up region and suppression of the spurious instability in the wake. It is clear from this figure that more particles are required to suppress the instabilities.

Fig. 12 shows that the spurious instability in the vortex layer is purely numerical since it can be delayed by increasing the precision in calculations. As one can see in this figure, the solution is free of instabilities when quadruple machine precision is employed. A convergence test performed for quadruple precision indicated that $p=75$ terms in the power series of the FMM for achieving machine error. These simulations are run for 200,000 particles and 375 steps using the RK4 with $\Delta t=8 \times 10^{-8}$. In the same figure, one can observe that quadruple precision does not improve the solution in terms of the roll-up process.

\subsection{Computational time}

The FMM provides two main benefits when coupled to the DVM. First, it provides a considerable reduction in the computational cost and, second, it allows simulations with larger numbers of particles and, therefore, higher resolution in the discretizations of the physical problems of interest. Here, we perform comparisons to quantify the savings in computational 

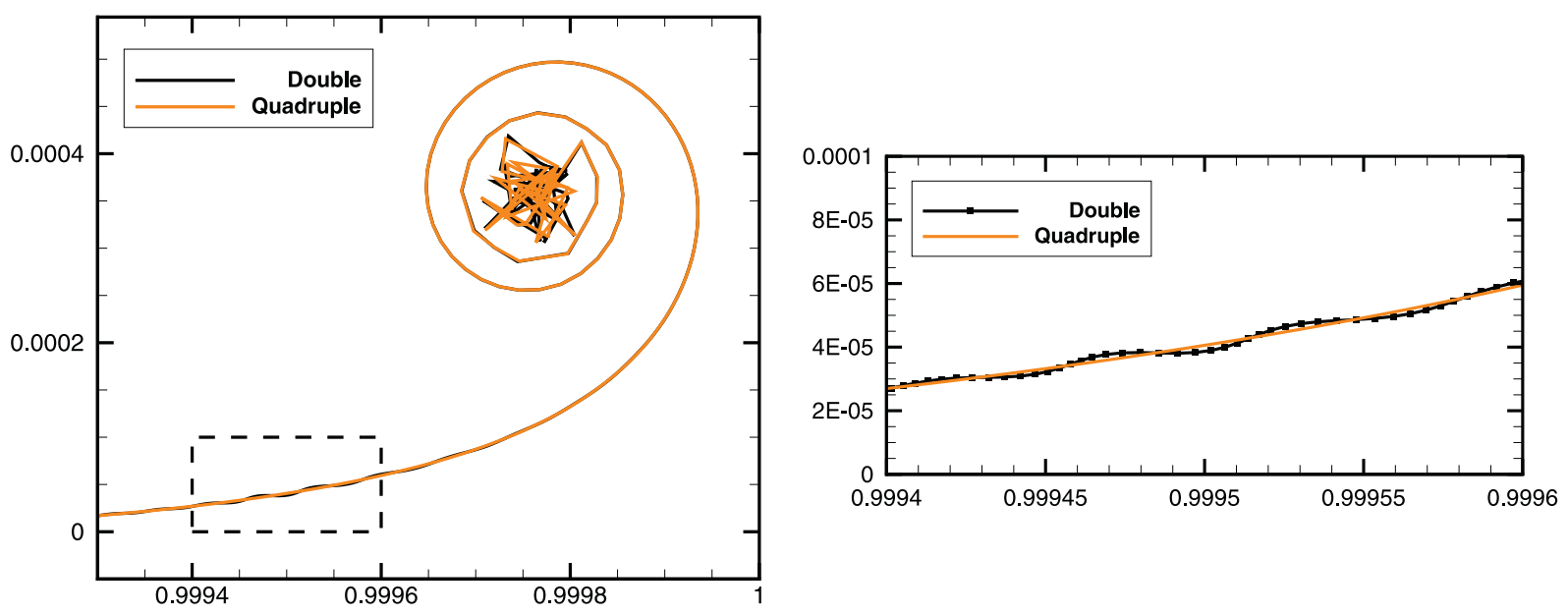

Fig. 12. Influence of machine precision on suppression of spurious instabilities for 200,000 vortex particles, $\sigma=1 \times 10^{-5}$, RK4, $\Delta t=8 \times 10^{-8}$ and $t=$ $3 \times 10^{-5}$.

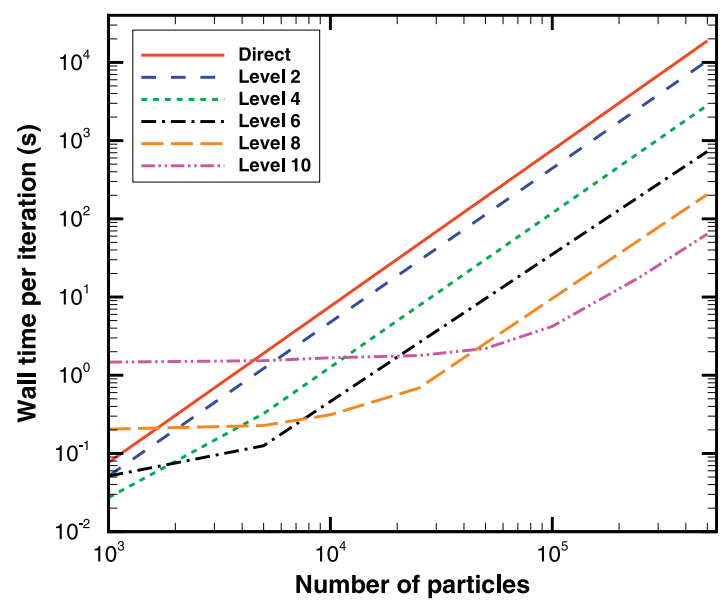

(a) Double precision.

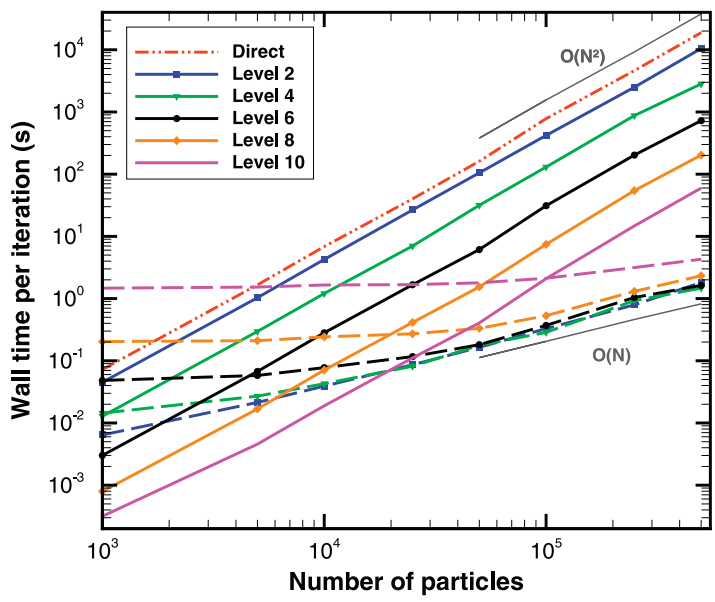

(b) Far-field and near-field operations.

Fig. 13. Computational time to evaluate the velocity field for Biot-Savart and FMM.

cost of the coupled FMM-DVM compared to the direct solution of the Biot-Savart law, which has a cost proportional to $\mathrm{O}\left(N^{2}\right)$. Comparisons are performed for a single evaluation of the velocity field induced by interactions among all vortex particles. An assessment of the computational cost is presented for different refinement levels and number of vortex particles in the wake discretization. All computations are performed using $p=40$ terms in the FMM series expansion, which guarantees computer accuracy in double precision. The current algorithm is written in FORTRAN language and simulations are performed in serial by a $2.5 \mathrm{GHz}$ Intel®Xeon ${ }^{\mathrm{TM}} \mathrm{E} 5-2670 \mathrm{v} 2 \mathrm{CPU}$.

Fig. 13(a) shows a comparison in terms of computational wall time for the direct evaluation of the Biot-Savart law and the FMM. It is possible to see that, for the number of particles analyzed, the latter provides faster solutions than the former, except for higher levels of refinement with a number of particles below 4000. Simulations are performed using up to $5 \times$ $10^{5}$ particles and, for this limit case, computational cost savings of 285 times are achieved by the fast multipole method. Also, it is interesting to notice that, for each level of refinement of the FMM, the computational cost is constant until a certain number of particles is reached. The fixed cost is dominant so higher plateaus are observed for finer maximum refinement levels. After the plateau, the cost behavior is also proportional to $\mathrm{O}\left(N^{2}\right)$ but higher refinement levels shift down the curves, reducing computational costs. This leads to intersections of the curves and, after a large enough number of elements, higher refinement levels are required to have faster simulations. This way, there is a best refinement level based on the number of elements in the discretization.

In order to understand the behavior of these curves, Fig. 13(b) shows a comparison between near-field and far-field costs of the FMM algorithm, given by solid and dashed lines, respectively. The total computational cost of the FMM is given by the sum of these two contributions and it should be of the form $\mathrm{O}\left(\mathcal{A}_{2} N^{2}+\mathcal{A}_{1} N+\mathcal{A}_{0}\right)$. The computational cost of the near-field 


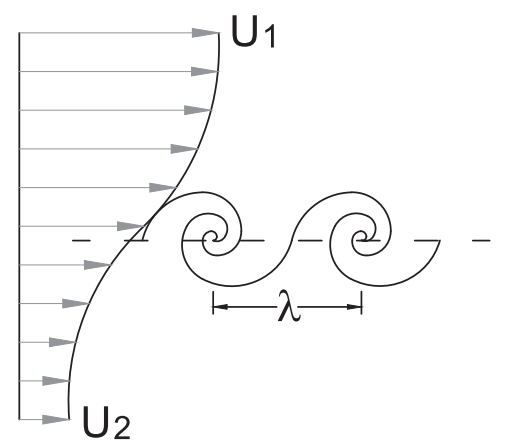

Fig. 14. Sketch of a periodic shear layer formation.

calculations is still $\mathrm{O}\left(\mathcal{A}_{2} N^{2}\right)$ since it represents the direct evaluations of the Biot-Savart law performed for vortex particles contained inside neighbor boxes of the FMM. For higher refinement levels, the near-field interaction cost is smaller since there are fewer direct evaluations at finer boxes, i.e., the coefficient $\mathcal{A}_{2}$ is reduced. This way, the cost of the direct evaluation is reduced. On the other hand, higher levels of refinement increase the coefficients $\mathcal{A}_{1}$ and $\mathcal{A}_{0}$ since more FMM operations are performed, which leads to higher far-field costs.

The maximum refinement level is then defined by the computational cost to evaluate a single time step due to the balancing of near and far-field operations. It is important to keep the refinement level in the range where the FMM computational cost is $\mathrm{O}\left(\mathcal{A}_{1} N+\mathcal{A}_{0}\right)$. However, one must not forget the requirement of Eq. (22) to guarantee that the size of the smallest box satisfies the Lamb-Oseen vortex criterion. Depending on the viscous core, this issue may keep the cost in the $\mathrm{O}\left(\mathcal{A}_{2} N^{2}\right)$ range. Finally, it is important to mention that adding an extra level of refinement increases the memory cost by a factor of 4 .

\section{Periodic shear layer}

The discrete vortex method can be employed to study the evolution of shear layers including the formation of the KelvinHelmholtz instability. In this case, the periodic boundary condition is implemented only in one dimension according to the physics of the problem, as shown in Fig. 14. With the DVM, a periodic shear layer can be discretized using $N$ vortex-particles and its temporal evolution can be simulated depending on the imposed initial conditions. The vorticity $\gamma$ of a continuous shear layer with length $\lambda$, with a jump in velocity $\Delta U$, is given by $\gamma=\Delta U \lambda$. Discretizing the shear layer using $N$ vortex elements with constant length $\frac{\lambda}{N}$ leads to $\Gamma_{k}=\frac{\Delta U \lambda}{N}$ and setting $\Delta U=1$ and $\lambda=1$, the circulation of each vortex is $\Gamma_{k}=\frac{1}{N}$.

If the periodic vorticity layer is initially straight, without perturbations, by symmetry of the problem, its self-induced velocity is null and there is no motion. However, computer truncation errors add a small random perturbation to the shear layer and spurious waves arise. In order to create a controlled solution, we add a sinusoidal displacement $y_{k}=A \sin \left(2 \pi x_{k}\right)$ along $-0.5<x_{k}<0.5$ with amplitude $A \ll 1$.

\subsection{Discrete vortex method for periodic problems}

The induced velocity at a point $z_{k}$ by a vortex-particle at $z_{j}$, with $\Gamma_{j}>0$ in the counter-clockwise sense, is given by the Biot-Savart law. If the vortex $j$ is replicated from $-\infty$ to $\infty$, with spatial periodicity $\lambda$, it is possible to calculate the influence of this array at $z_{k}$ according to

$$
w_{k}=\sum_{n=-\infty}^{\infty} \mathrm{i} \frac{\Gamma_{j}}{2 \pi}\left(\frac{1}{z_{k j}+n \lambda}\right) \text {. }
$$

For $\lambda=1$, the summation inside parentheses has an exact solution given by the cotangent function (see Abramowitz and Stegun [38])

$$
\pi \cot \left(\pi z_{k j}\right)=\frac{1}{z_{k j}}+\sum_{n=1}^{\infty}\left(\frac{1}{z_{k j}-n}+\frac{1}{z_{k j}+n}\right) .
$$

This solution can be desingularized using an array of Lamb-Oseen vortex particles, written as

$$
w_{k}=\mathrm{i} \frac{\Gamma_{j}}{2 \pi}\left\{\pi \cot (\pi z)-\sum_{n=-1}^{1}\left(\frac{1}{z_{k j}+n}\right) \exp \left[-\frac{\left(x_{k j}+n\right)^{2}+\left(y_{k j}\right)^{2}}{\sigma^{2}}\right]\right\}
$$




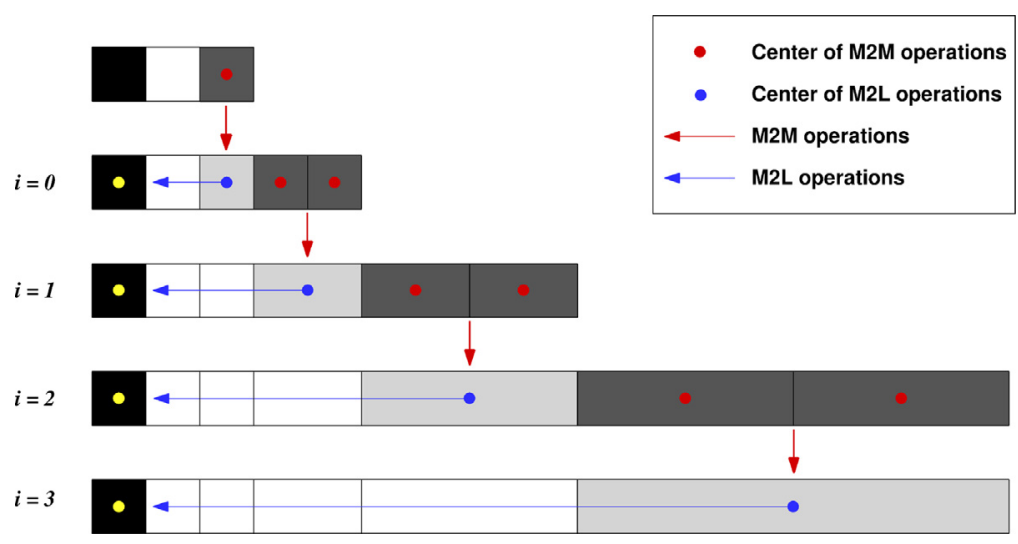

Fig. 15. Optimized scheme for far-field interactions of clusters of boxes $\mathcal{P}$.

\subsection{Fast multipole method for periodic problems}

Fast multipole methods can be implemented for the solution of three-dimensional problems where all directions require periodic boundary conditions. For example, a cubical box with isotropic turbulence would require such implementation. Here, we analyze a physical problem which has only one periodic direction and, hence, the implementation of periodic boundary conditions in the FMM are explained in one dimension. The aim of this work is to discuss about the numerical errors that appear due to the implementation of periodic boundary conditions and its effects on inviscid flows. In this context, we propose a novel approach that reduces the numerical errors from the implementation of the periodic FMM, and that can be generalized for multiple periodic dimensions if necessary.

In a one dimensional problem, the induced velocity of an infinite array of vortex particles with spatial periodicity $\lambda=1$ is given by Eq. (24). From this equation, the convolution kernel is

$$
\sum_{n=-\infty}^{-2}\left(\frac{1}{z_{k j}+n}\right)+\left(\frac{1}{z_{k j}-1}\right)+\left(\frac{1}{z_{k j}}\right)+\left(\frac{1}{z_{k j}+1}\right)+\sum_{n=2}^{\infty}\left(\frac{1}{z_{k j}+n}\right),
$$

where each term $n$ in the previous summation represents a periodic box, at level $\ell=0$, in the infinite array.

The divide-and-conquer strategy of the FMM can be explored to reduce the interaction costs among clusters at level 0 , and, to do so, an additional upward pass using the M2M computation can be employed. The basis of this optimized scheme is that periodic boxes $|n| \geq 2$ can be further grouped to create larger clusters as shown in Fig. 15 . In this figure, this procedure is shown from periodic level $i=0$ up to the illustrative maximum level $I=3$. There, the red arrows indicate the results from $\mathrm{M} 2 \mathrm{M}$ clustering operations, while the red dots (online version in color) are the center of the dark gray boxes to be clustered. In the periodic FMM, the M2M step is still computed by Eq. (12), modified to account for 2 smaller boxes instead of the 4 boxes used in the free-domain FMM. These 2 boxes from periodic level $i-1$ can be clustered at level $i$, for $i \geq 1$, using

$$
\mathcal{M}_{k}^{(i)}=\sum_{m=1}^{k}\left[\mathcal{M}_{m}^{(i-1)}\left(\begin{array}{c}
k-1 \\
m-1
\end{array}\right)\left(z_{i-1, b 1}^{k-m}+z_{i-1, b 2}^{k-m}\right)\right]-\mathcal{M}_{0}^{(i-1)} \frac{\left(z_{i-1, b 1}^{k}+z_{i-1, b 2}^{k}\right)}{k} .
$$

In the above equation, the distances in the complex plane from the center of the two clusters at level $i-1$ to the new cluster center at level $i$ are, respectively, $z_{i-1, b 1}=-2^{i-2}$ and $z_{i-1, b 2}=+2^{i-2}$. The superscripts inside parenthesis indicate levels of refinement in the periodic FMM, as shown in Fig. 15, and the other superscripts are exponents. The periodic level $i=0$ coincides with the free-domain level $\ell=0$ and so $\mathcal{M}_{0}^{(0)}$ is the same for both schemes. For levels $i>0$, it is given by

$$
\mathcal{M}_{0}^{(i-1)}=2^{i-1} \mathcal{M}_{0}^{(0)}
$$

One must note that M2M operations are performed only once for both left and right sides of the central domain since the multipole expansions are identical for both sides.

The next step corresponds to the computation of the M2L pass for the periodic boxes. For these calculations, from the separation criterion [8], it is only mandatory that the clusters are separated by at least the size of the source-boxes. In Fig. 15, the blue arrows represent the M2L operations from the center of the larger source-clusters indicated by blue dots and light gray boxes. The M2L operations must be performed twice to account for both left and right sides of the central domain. One must not forget that due to the separation criterion, the immediate neighbors $|n|=1$ of the central domain only perform M2L operations in a similar fashion to the free domain FMM, i.e., using smaller boxes at levels $\ell \geq 1$. This way, their interactions are not shown in Fig. 15. Hence, the M2L computations for the periodic boxes are performed directly to 


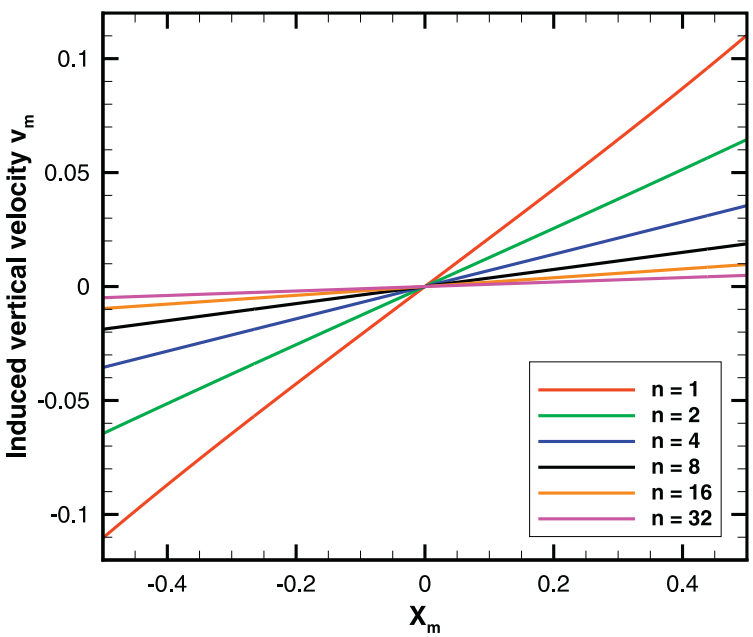

(a) Spurious precession velocity based on the number of domains, $n$.

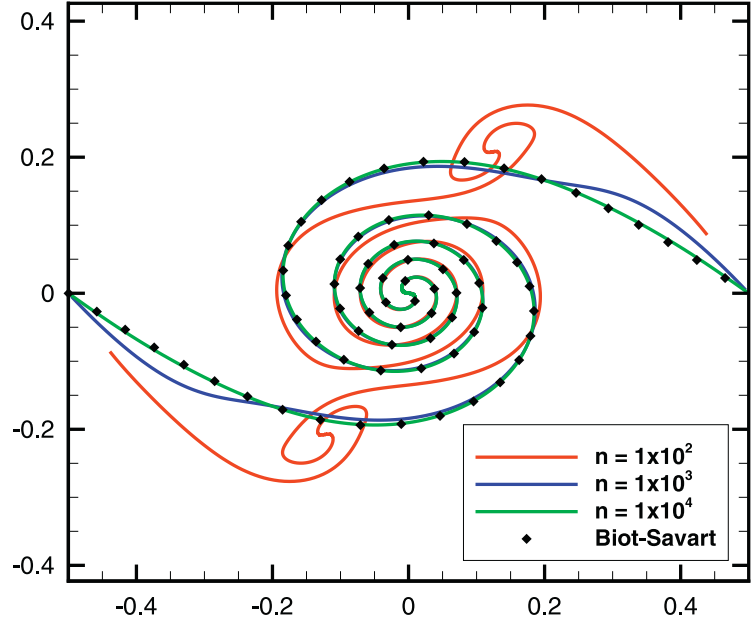

(b) Solution of a shear-layer with spurious precession velocity.

Fig. 16. Influence of number of domains in periodicity for a finite shear layer discretized by 5120 vortex particles, $A=0.01, \sigma=0.05$, RK4 with $\Delta t=0.01$ until $t=2.0$.

the central domain for all levels $i=0$ to $I$, for $m=1$ to $p$, as

$$
\mathcal{L}_{m}^{(\mathrm{M} 2 L)}=\sum_{i=0}^{I} \sum_{s=1}^{2}\left\{-\frac{\mathcal{M}_{0}^{(i)}}{m \cdot z_{i, s}^{m}}+\frac{1}{z_{i, s}^{m}} \sum_{k=1}^{p}\left[\mathcal{M}_{k}^{(i)}\left(\begin{array}{c}
m+k-1 \\
k-1
\end{array}\right) \frac{(-1)^{k}}{z_{i, s}^{k}}\right]\right\} .
$$

In this equation, the distance from the left and right clusters with respect to the central domain are given by $z_{i, 1}=+(1.5 \times$ $\left.2^{i}+0.5\right)$ and $z_{i, 2}=-\left(1.5 \times 2^{i}+0.5\right)$, respectively.

\subsection{Convergence of the periodic FMM}

The FMM is applied to accelerate the solution of a periodic shear layer. The method is based on a series expansion of the cotangent function, which governs the vortex dynamics in a periodic layer, and it may be truncated in $p$ terms. The convergence study presented in Fig. 7 for the free-domain FMM shows that machine precision is achieved for about $p=40$ terms and so, this value is used in all simulations using the FMM in this section. Also, a source of error of the periodic FMM comes from the replication of domains and it arises from the truncation of an infinite shear layer. Each domain represents a small shear layer that works as a boundary condition for the central domain. For a distribution of $N$ vortex particles, there is an asymmetric distribution of elements around an observer $k$. This unbalancing induces a higher vertical velocity at $k$, and it is expected to be higher if $k$ is further away from the center of the shear layer. The unbalancing moves the particles such that the wake tips begin to roll-up. Also, the entire wake has a precession movement about its center $x_{C}=\frac{\left(x_{N}+x_{1}\right)}{2}$. Adding $n$ domains to both tips, i.e., going from a free-domain layer to a periodic layer, the asymmetry of particles are moved further away, which reduces the induced precession velocity, $v_{k}$, evaluated by $v_{k} \sim \mathrm{O}\left(\frac{2 k-N-1}{N} \frac{1}{n}\right)$ with an error decay based on the number of domains about $\mathrm{O}\left(\frac{1}{n}\right)$.

The measurements in Fig. 16 are performed for 5120 vortex particles in the first time step of a undisturbed shear layer, where it is expected from a delicate balance that the induced vertical velocity is null, as described by Lewis [35]. It confirms that a vortex close to the center has a smaller precession velocity while one located towards the wake tip has a larger velocity, as shown in Fig. 16(a). In the case of an initially perturbed shear layer with sinusoidal shape, the problem is more complex. The results for long-time integrations are shown in Fig. 16(b), where the use of additional domains reduce both precession and secondary roll-up. It is possible to see a convergence of the FMM scheme to the solution of the Biot-Savart law using the cotangent function. The present numerical solution is obtained using 5120 vortex particles with viscous core $\sigma=0.05$ with the RK4 scheme and $\Delta t=0.01$ until $t=2.0$. The initial amplitude of the sinusoidal displacement is $A=0.01$.

Since this spurious precession is purely due to the unbalancing from truncation of the periodic shear layer, one must overcome its presence. To do so, $N-k$ vortex particles with intensity $\Gamma=\frac{1}{N}$ are placed at the left side of a vortex $k$ in the $n+1$ domain, i.e., after the last domain $n$. Similarly, another set of $k-1$ particles are placed in the $n+1$ domain to the right of the vortex $k$. This balancing procedure eliminates any precession in a horizontal, undisturbed shear layer since all vortex particles have null velocity and any error is purely due to the machine precision. However, a perturbed layer no longer has symmetry inside the central domain and it also has the $u$ component of the velocity. This way, compared to the cotangent solution, there is still an error for the induced velocity proportional to the number of periodic images at both sides of the 


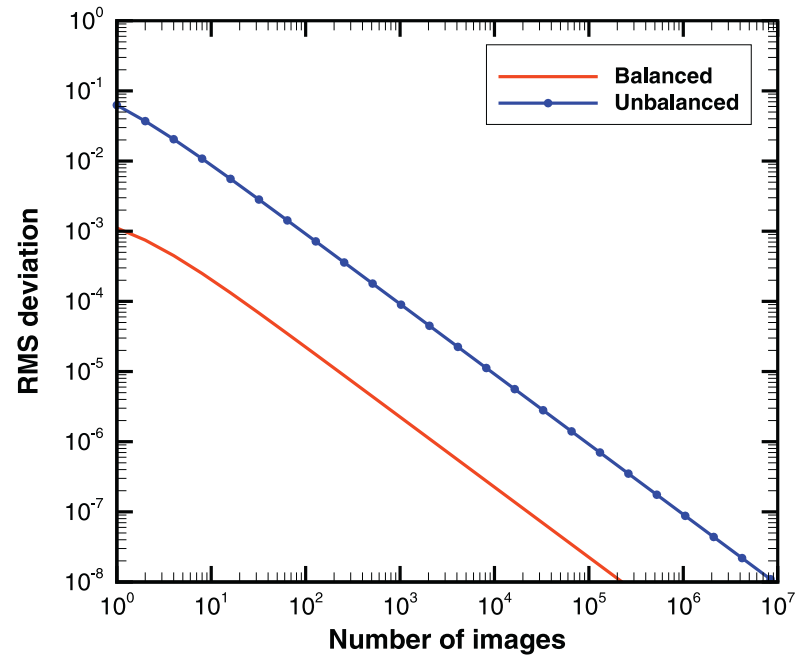

(a) Rate of convergence for the periodic FMM with and without balancing.

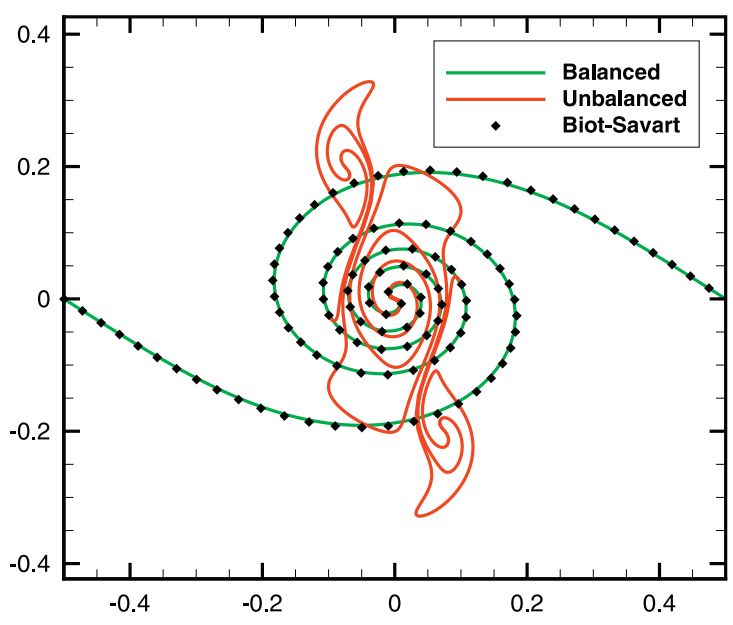

(b) Influence of balance for $n=10$ periodic domains representing a finite shear layer.

Fig. 17. Error reduction when using a balanced shear layer.

central domain. Fig. 17(a) presents the RMS deviation calculated with 5120 vortex particles only for the first step of the simulation. It is possible to see that the proposed balancing reduces the error and accelerates the convergence based on the number of periodic domains.

The use of a shear layer balancing is more than just error-reducing. In Fig. 17(b), for only 10 replicated domains, the result with balancing is closer to the Biot-Savart law solution using a Lamb-Oseen vortex array, Eq. (26). The unbalanced solution not only has precession but also secondary roll-ups, leading to a spurious solution. In this figure, the results are obtained using the same parameters as those for Fig. 16 .

Fig. 18(a) presents a finite shear layer without the balancing that enforces the periodic boundary conditions. Since balancing is necessary, the FMM can be further modified to consider the additional particles and to avoid excessive computational cost. The whole balancing procedure is explained in Fig. 18(b-e). The main idea is to cluster, at the left, the particles indicated by $k+1$ to $N$, while at the right side, the cluster is from 1 to $k-1$ (see Fig. 18(b)). The clustering uses the existent multipole expansions from the free-domain FMM for levels $\ell=1$ to $L-1$. The boxes which are clustered are those in the inner portion of the wake shown in light gray in Fig. 18(c). If any of the white boxes appearing in this previous figure is used, unbalancing will occur. In the procedure, two new equivalent clusters at the boundaries of the truncated domain are created in the red dots, as presented in Fig. 18(d). These two new clusters interact directly with the smallest boxes (dark gray in the figure), in the central domain, at level $L$. These calculations use only M2L operations with no further L2L computations. For a precise balancing, the particles inside both replicated finer boxes in the boundaries, at level $L$, interact with the dark gray boxes directly using the Biot-Savart law. Hence, a new $\mathrm{O}\left(\tilde{\mathrm{N}}^{2}\right)$ cost is added where $\tilde{\mathrm{N}}$ is the number of particles in the column of the finest level boxes (Fig. 18(e)).

The convergence based on the number of domains, $n$, of the solution with balancing is investigated for long-time integrations. The computation of the FMM periodicity uses the optimized fast summation of the far-field previously described. The solutions maintain the correct shear layer pattern independently of the number of domains employed. The calculations are performed with the same parameters as in Fig. 16.

The solution of the periodic shear layer also presents spurious instabilities similar to those from the Trefftz plane when $\sigma \rightarrow 0$. A similar procedure as that presented in the previous section can be applied to solve such issues and more details can be found in Ricciardi [39].

\subsection{Computational time}

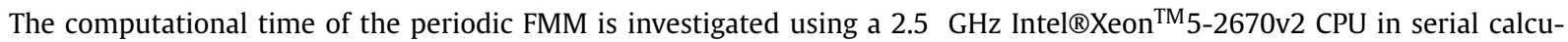
lations. In the periodic FMM, two new costs must be analyzed: that from balancing as well as that from the level 0 periodicity from inclusion of the $n$ image boxes. A comparison of total cost from direct evaluation using the Biot-Savart cotangent solution with the periodic FMM is performed up to $4 \times 10^{5}$ vortex elements and the results are shown in Fig. 20(a). The fixed cost of the FMM is relevant for a small number of particles, however, the cost reduction using the periodic FMM is about 480 times that of the direct summation for the maximum number of particles investigated. 


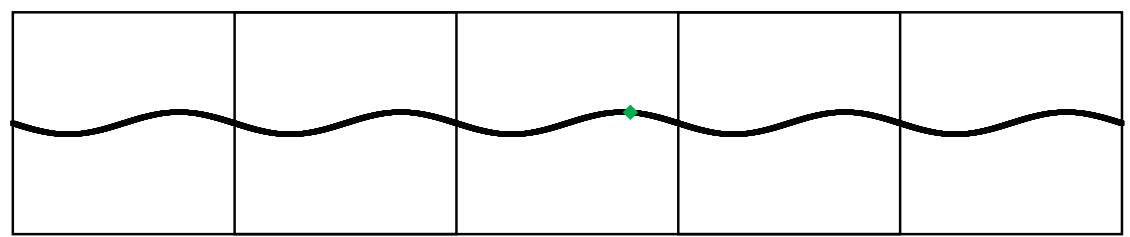

(a) Periodicity of the FMM without balancing. Here, the periodicity is considered with respect to the green vortex particle in the central domain.

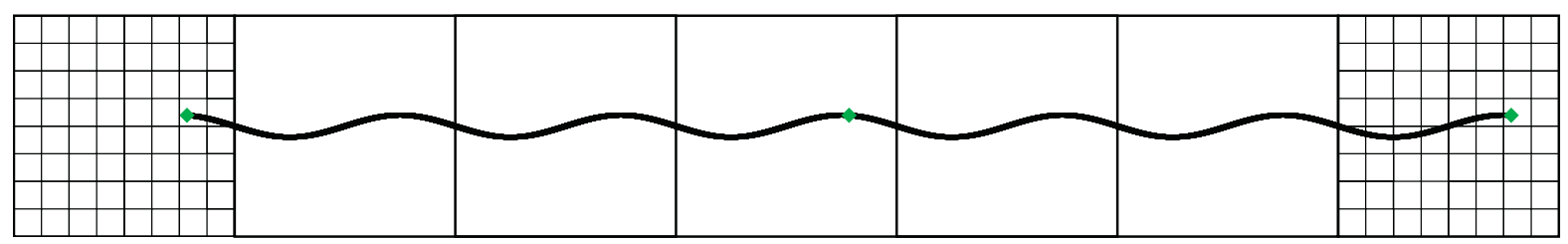

(b) Creation of boxes at the finest level in both boundaries of the periodicity.

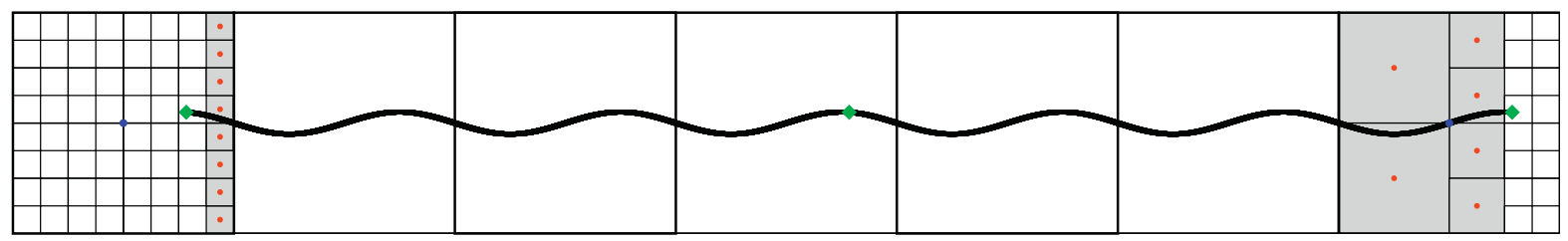

(c) M2M operations to level 0 of the boxes in the internal part of the wake (red dots to blue dots).

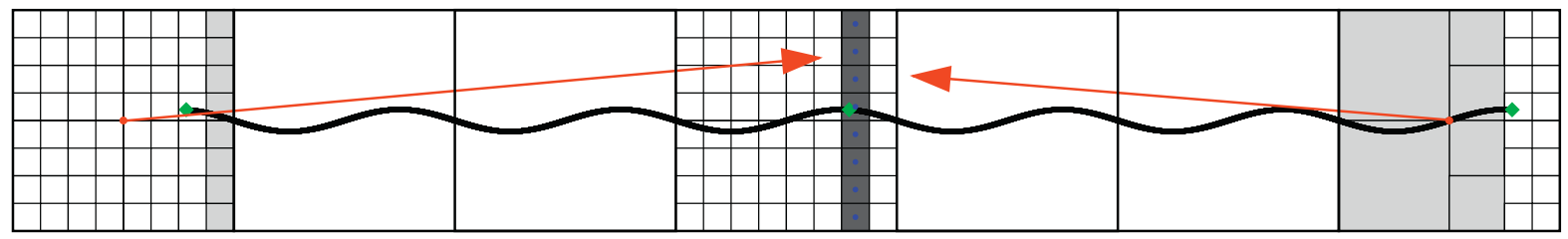

(d) M2L operations using the multipole expansions at both tips of the wake (red dots to blue dots in dark gray boxes).

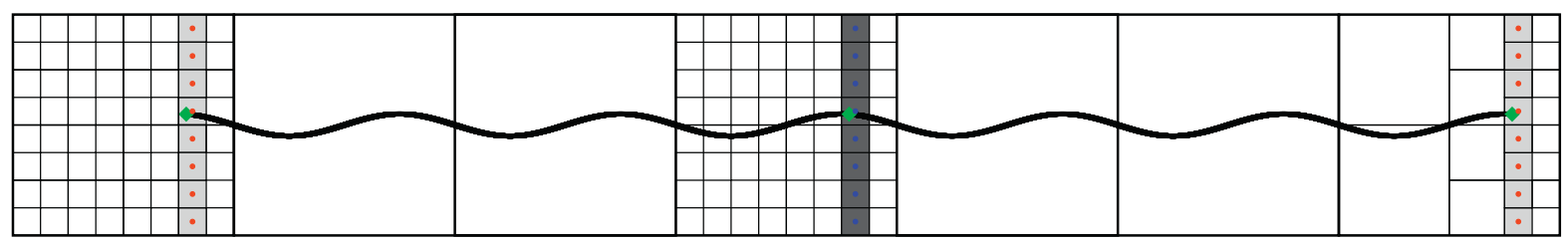

(e) Direct interactions in the smaller boxes coincident with the replica of the main particle (particles inside boxes with red dots to green particle in the central domain).

Fig. 18. Current implementation of the wake balance procedure using the FMM. 


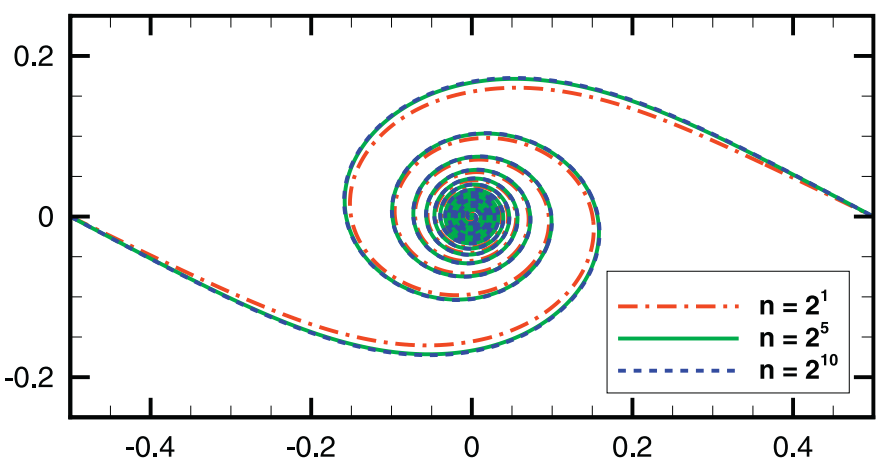

Fig. 19. Effects of the number of domains in the solution of the periodic FMM using 5120 vortex, $A=0.005, \sigma=0.02$, RK 4 with $\Delta t=0.01$ until $t=2.0$.

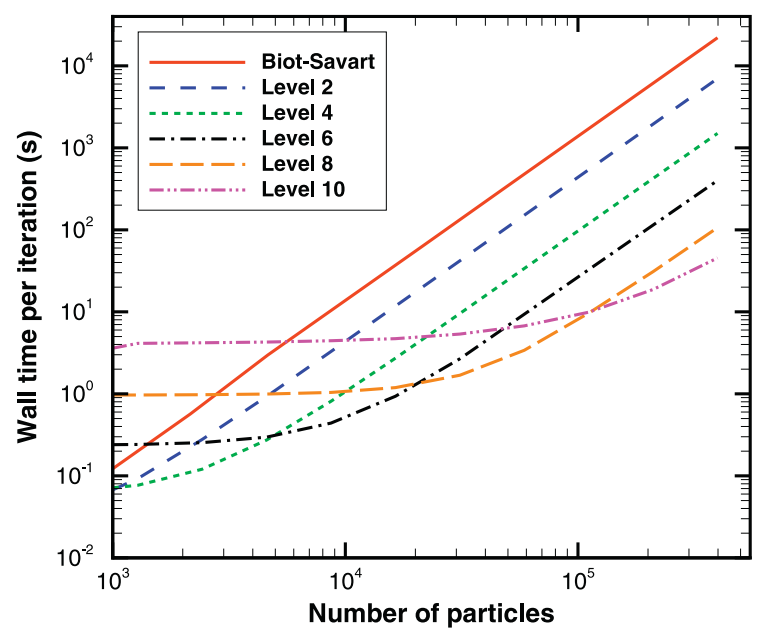

(a) Total computational time per iteration.

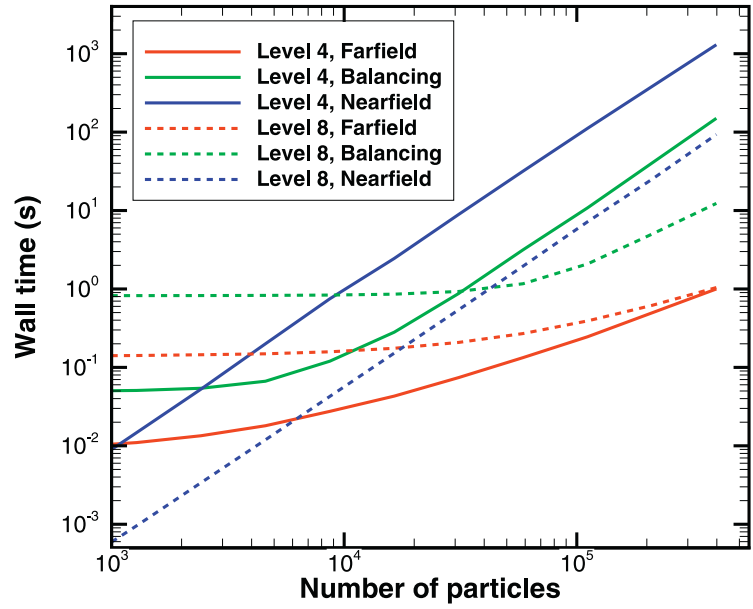

(b) Computational time of different operations.

Fig. 20. Computational time for a single evaluation of the periodic FMM.

The balancing cost of the periodic FMM is shown in Fig. 20(b), where the red and blue lines represent the far-field and near-field operations of the free-domain FMM, respectively. The green lines represent the overall costs of the proposed periodic implementation which include the steps from Fig. 18(c-e). This new cost is compared to the near-field computations inside the central domain. Although a new $\mathrm{O}\left(N^{2}\right)$ cost is added, this operation is nearly $10 \%$ of the existing near-field cost for large $N$ and it does not compromise the use of wake balancing. Moreover, this cost is small since, in the present case, the wake is concentrated in the center of the domain and only a few boxes are non-empty. In a problem with a homogeneous distribution of particles along the entire FMM domain one may expect an increased balancing cost.

\section{Conclusions}

Lagrangian simulations of unsteady vortical flows are performed using the discrete vortex method, DVM, accelerated by the multi-level fast multipole method, FMM. The combination of the FMM-DVM algorithms is discussed for free domain and periodic problems with focus on implementation details to reduce numerical dissipation and to avoid spurious solutions in unsteady inviscid flows. In the current work, we provide an assessment of the parameters which control the errors and the numerical dissipation of the coupled DVM-FMM for the solution of the Trefftz plane problem, which models the wing tip vortex formation in aircraft, and for a periodic shear layer.

It is shown that the vortex regularization model and the time marching scheme introduce numerical dissipation to the convective operator of the DVM. A higher-order time marching scheme and a small viscous core in the vortex model are required to control the numerical dissipation. Spurious numerical instabilities may arise due to the lack of diffusion in the convective operator of the DVM. In order to obtain accurate solutions which delay the formation of secondary roll-ups in the FMM-DVM coupling, a large number of truncation terms in the FMM series must be employed in the calculations. As expected, increasing the depth of the tree in the FMM is shown to reduce the overall computational cost of the method. 
However, the increase of this level is limited by the length of the vortex core in the regularization model. The FMM allows simulations with higher resolution in the wake discretizations and, therefore, it improves the solutions delaying the formation of spurious numerical instabilities.

The evolution of a temporal shear layer is obtained by a periodic FMM implementation. We present a novel approach to increase the accuracy of periodic flow calculations using the DVM-FMM. This approach avoids a spurious precession of the periodic shear layer and solutions are shown to converge to the direct Biot-Savart calculation using a cotangent function. The overall computational cost of the periodic FMM is shown to be similar to that of the free-domain FMM. The approach can be also applied for multiple periodic directions in three-dimensional flows.

\section{Acknowledgements}

The authors acknowledge the financial support received from Fundação de Amparo à Pesquisa do Estado de São Paulo, FAPESP, under Grants No. 2013/03413-4 and 2013/07375-0 and from Conselho Nacional de Desenvolvimento Científico e Tecnológico, CNPq, under Grants No. 470695/2013-7 and 305277/2015-4. The authors also acknowledge CAPES for providing a MSc. scholarship to the first author. The computational resources provided by CENAPAD-SP under Project No. 551 are also acknowledged.

\section{References}

[1] Chorin AJ. Numerical study of slightly viscous flow. J Fluid Mech 1973;57:785-96

[2] Hammer P, Altman A, Eastep F. Validation of a discrete vortex method for low reynolds number unsteady flows. AIAA J 2014;52:643-9.

[3] Ramesh K, Gopalarathnam A, Granlund K, Ol MV, Edwards JR. Discrete-vortex method with novel shedding criterion for unsteady aerofoil flows with intermittent leading-edge vortex shedding. J Fluid Mech 2014;751:500-38.

[4] Quinn DB, Moored KW, Dewey PA, Smits AJ. Unsteady propulsion near a solid boundary. J Fluid Mech 2014;742:152-70.

[5] Dynnikova GY, Dynnikov YA, Guvernyuk SV. Mechanism underlying kármán vortex street breakdown preceding secondary vortex street formation. Phys Fluids 2016;28:054101.

[6] Barba LA, Leonard A, Allen CB. Advances in viscous vortex methods - eshless spatial adaption based on radial basis function interpolation. Int J Numer Methods Fluids 2004;46:001-32.

[7] Aref H. Integrable, chaotic, and turbulent vortex motion in two-dimensional flows. Annu Rev Fluid Mech 1983;15:345-89.

[8] Greengard L, Rokhlin V. A fast algorithm for particle simulations. J Comput Phys 1987;73:325-48.

[9] Carrier J, Greengard L, Rokhlin V. A fast adaptive multipole algorithm for particle simulations. SIAM Journal on Scientific and Statistical Computing 1988;9:669-86

[10] Wolf WR. Airfoil aeroacoustics: Les and acoustic analogy. Ph.D. thesis. Stanford University; 2011.

[11] Darve E. The fast multipole method: numerical implementation. J Comput Phys 2000;160:195-240.

[12] Darve E, Havé P. Efficient fast multipole method for low-frequency scattering. J Comput Phys 2004;197:341-63.

[13] Gumerov NA, Duraiswami R. Fast multipole methods for the helmholtz equation in three dimensions. Elsevier; 2005.

[14] Wolf WR, Lele SK. Acoustic analogy formulations accelerated by fast multipole method for two-dimensional aeroacoustic problems. AIAA J 2010;48:2274-85.

[15] Wolf WR, Lele SK. Aeroacoustic integrals accelerated by fast multipole method. AIAA J 2011;49:1466-77.

[16] Gumerov NA, Duraiswami R. Efficient FMM accelerated vortex methods in three dimensions via the lamb-helmholtz decomposition. J Comput Phys 2013;240:310-28.

[17] Lambert CG, Darden TA, Board Jr JA. A multipole-based algorithm for efficient calculation of forces and potentials in macroscopic periodic assemblies of particles. J Comput Phys 1996;126:274-85.

[18] Gumerov NA, Duraiswami R. A method to compute periodic sums. J Comput Phys 2014;272:307-26.

[19] Yokota R, Barba LA. Fmm-based vortex method for simulation of isotropic turbulence on gpus, compared with a spectral method. Comput Fluids 2013;80:17-27.

[20] Yokota R, Obi S. Comparing vortex methods and finite difference methods in a homogeneous shear flow. Int J Numer Methods Fluids 2010;63:828-46.

[21] Sakajo T, Okamoto H. An application of draghicescu's fast summation method to vortex sheet motion. J Phys Soc Jpn 1997;67:462-70.

[22] Draghicescu CI, Draghicescu M. A fast algorithm for vortex blob interactions. J Comput Phys 1995;116:69-78.

[23] Marple GR, Barnett A, Gillman A, Veerapaneni S. A fast algorithm for simulating multiphase flows through periodic geometries of arbitrary shape. SIAM J Sci Comput 2016;38:740-72.

[24] Smith JHB. Vortex flows in aerodynamics. Annu Rev Fluid Mech 1986;18:221-42.

[25] Murray SD, White SDM, Blondin JM, Lin DNC. Dynamical instabilities in two-phase media and the minimum masses of stellar systems. Astrophys J $1993 ; 407: 588-96$

[26] Smyth WD, Moum JN. Ocean mixing by kelvin-helmholtz instability. Oceanography 2012;25:140-9.

[27] Herrmann M. An eulerian level set/vortex sheet method for two-phase interface dynamics. J Comput Phys 2005;203:539-71.

[28] Spalart PR. Vortex methods for separated flows. Tech. Rep., 100068. NASA Ames Research Center; 1988.

[29] Fink PT, Soh WK. A new approach to roll-up calculations of vortex sheets. Proc R S London 1978;362:195-209.

[30] Krasny R. A study of singularity formation in a vortex sheet by the point-vortex approximation. J Fluid Mech 1986a;167:65-93.

[31] Krasny R. Desingularization of periodic vortex sheet roll-up. J Comput Phys 1986b;65:292-313.

[32] Krasny R. Computation of vortex sheet roll-up in the trefftz plane. J Fluid Mech 1987;184:123-55.

[33] Abid M, Verga A. Stability of a vortex sheet roll-up. Phys Fluids 2002;14:3829-34.

[34] Holm DD, Nitsche M, Putkaradze V. Euler-alpha and vortex blob regularization of vortex filament and vortex sheet motion. J Fluid Mech 2006;555:149-76

[35] Lewis RI. Vortex element method for fluid dynamic analysis of engineering systems. Cambrigde, England, U.K.: Cambridge Univ. Press; 1991.

[36] Nishimura N. Fast multipole accelerated boundary integral equation methods. Appl Mech Rev 2002;55:299-324.

[37] Hamilton JT, Majda G. On the Rokhlin-Greengard method with vortex blobs for problems posed in all space or periodic in one direction. J Comput Phys 1995;121:29-50.

[38] Abramowitz M, Stegun IA. Handbook of mathematical functions with formulas, graphs and mathematical tables. U.S.A.: National Bureau of Standards; 1964.

[39] Ricciardi TR. Fast multipole discrete vortex method applied to unsteady flow simulations. MSc thesis: University of Campinas; 2016. 